\title{
Wavelength-Selective Uncaging of Two Different Photoresponsive Groups on One Effector Molecule for Light-Controlled Activation and Deactivation
}

Isam Elamrit, ${ }^{[a]}$ Chahinez Abdellaouit ${ }^{[b]}$ Jasleen Kaur Bains, ${ }^{[a]}$ Katharina Felicitas Hohmann ${ }^{[a]}$ Santosh Lakshmi Gande, ${ }^{[a]}$ Elke Stirnal, ${ }^{[a]}$ Josef Wachtveitl*, ${ }^{[b]}$ and Harald Schwalbe*[a] Institute of Organic Chemistry and Chemical Biology, Goethe-University, Frankfurt am Main, Germany Institute of Physical and Theoretical Chemistry, Goethe-University, Frankfurt am Main, Germany $\$$ These authors contributed equally in this work

Correspondence to: wveitl@theochem.uni-frankfurt.de

\section{Contents}

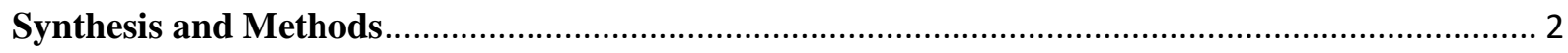

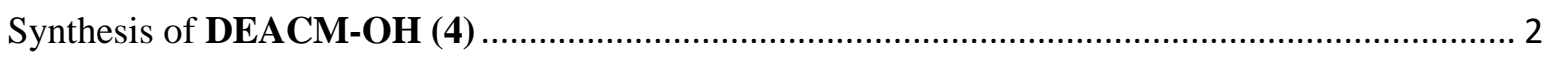

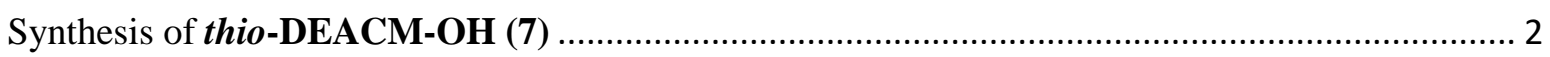

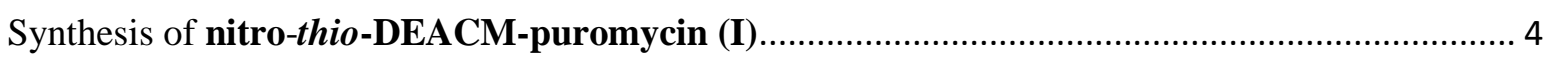

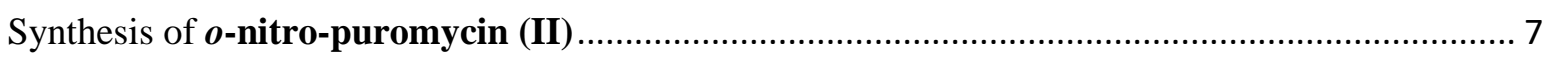

Synthesis of substituted 4-hydroxy-coumarylmethyl-derivatives ............................................. 10

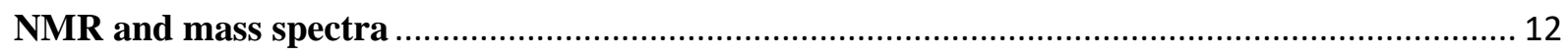

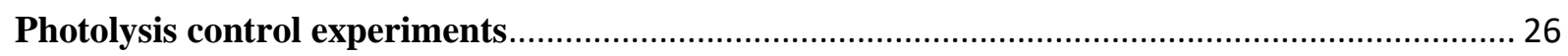

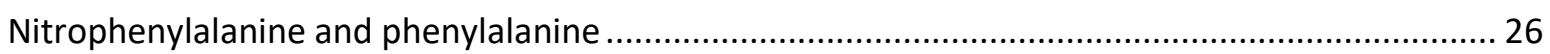

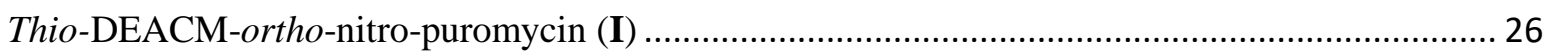

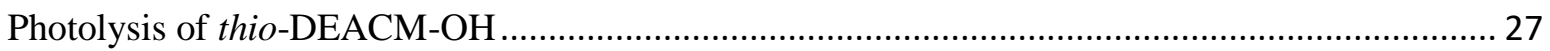

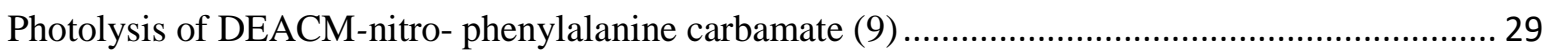

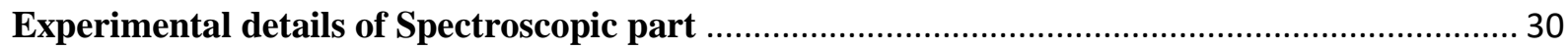

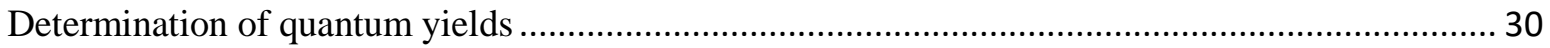

Absorptions spectra of the doubly caged compound thio-DEACM-ortho-nitro-puromycin and its

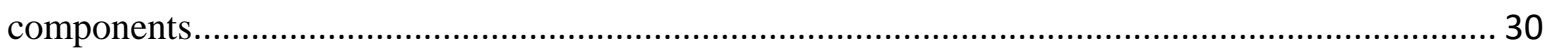

Investigation of thio-DEACM cage and its photolysis products ...................................................... 31

Spectral changes of nitro-puromycin upon illumination ............................................................... 32

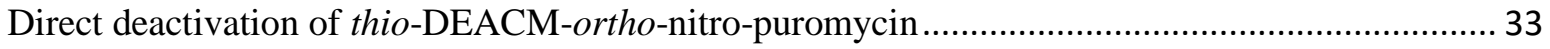

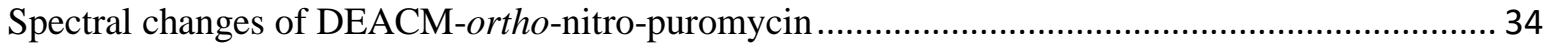

Spectral changes of thio-DEACM-ortho-nitro-puromycin in methanol / water ................................ 35 


\section{Synthesis and Methods}

\section{Synthesis of DEACM-OH (4)}<smiles>CCN(CC)c1ccc2c(C)cc(=O)oc2c1</smiles>

1

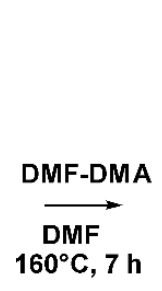<smiles>CCN(CC)c1ccc2c(/C=C/N)cc(=O)oc2c1</smiles>
$\underset{\mathrm{RT}, 5 \mathrm{~h}}{\stackrel{\mathrm{THF} / \mathrm{H}^{2}}{\longrightarrow}}$<smiles>CCN(CC)c1ccc2c(C=O)cc(=O)oc2c1</smiles>

$\stackrel{\mathrm{NaBH}_{4}}{\longrightarrow}$ RT, 4h<smiles>CCN(CC)c1ccc2c(CO)cc(=O)oc2c1</smiles>

Synthesis Procedure and Characterization data of DEACM-OH 4 can be found in our recent publications. ${ }^{[1,2]}$

Synthesis of thio-DEACM-OH (7)

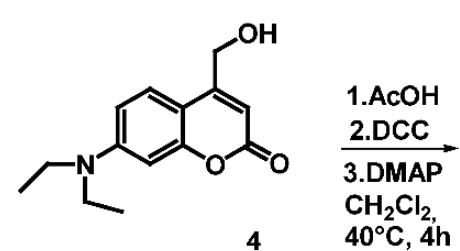

4<smiles>CCN(CC)c1ccc2c(COC(C)O)cc(=O)oc2c1</smiles>

5

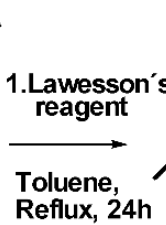

Reflux, 24h

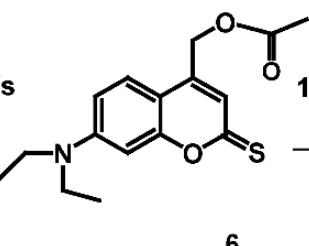

6

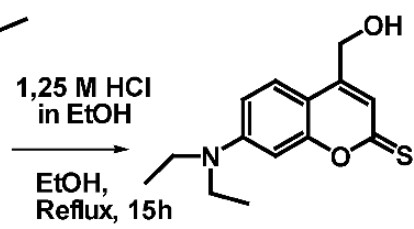

Reflux, 15h

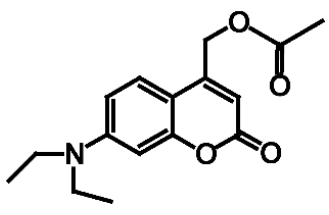

DEACM-AA (5)

7-(diethylamino)-2-oxo-2H-chromen-4-yl)methyl acetate

Compound 4 (1g, 4 mmol, 1 eq.) was solved in dry dichloromethane $(50 \mathrm{~mL})$ and acetic acid $(276 \mu \mathrm{l}$, $4.8 \mathrm{mmol}, 1.2 \mathrm{eq}$.) and 4-(imethylamino)pyridine (0,6 g, $4.8 \mathrm{mmol}, 1.2 \mathrm{eq}$.) were added and cooled down to $0^{\circ} \mathrm{C}$ under nitrogen and stirred for $15 \mathrm{~min}$. Than 1,3-dicyclohexylcarbodiimide $(1 \mathrm{~g}, 4.8 \mathrm{mmol}, 1.2$ 
eq.) was added and stirred at room temperature in dark hood for $10 \mathrm{~h}$. The reaction mixture was washed with $1.2 \mathrm{M} \mathrm{HCl}(3 \times 100 \mathrm{~mL})$ and $50 \mathrm{~mL} \mathrm{NaHCO}_{3}(3 \times 100 \mathrm{~mL})$ and dried over $\mathrm{MgSO}_{4}$ and lyophilized to yield a yellow solid.

Reaction yield: $63.7 \mathrm{mg}(0.22 \mathrm{mmol}, 54.3 \%)$.

$\mathbf{R}_{\mathbf{f}}: 0.79$ (DCM/MeOH. 95/5)

${ }^{1} \mathbf{H}$ NMR $\left(\mathrm{CDCl}_{3}, 600 \mathrm{MHz}\right): \delta=7.24\left(\mathrm{~d},{ }^{3} J(\mathrm{H}, \mathrm{H})=9.0 \mathrm{~Hz}, 1 \mathrm{H}\right), 6.59\left(\mathrm{dd},{ }^{3} J(\mathrm{H}, \mathrm{H})=9.0 \mathrm{~Hz}, 1 \mathrm{H}\right), 6.50$ $(\mathrm{s}, 1 \mathrm{H}), 6.10(\mathrm{~s}, 1 \mathrm{H}), 5.15(\mathrm{~s}, 2 \mathrm{H}), 3.35\left(\mathrm{q},{ }^{3} J(\mathrm{H}, \mathrm{H})=7.1 \mathrm{~Hz}, 4 \mathrm{H}\right), 2.12(\mathrm{~s}, 3 \mathrm{H}), 1.21\left(\mathrm{t},{ }^{3} J(\mathrm{H}, \mathrm{H})=7.1\right.$ $\mathrm{Hz}, 6 \mathrm{H}) \mathrm{ppm}$.

MS (ESI): $\mathrm{m} / \mathrm{z}$ calcd for $\mathrm{C}_{16} \mathrm{H}_{19} \mathrm{NO}_{4}: 312.12[M+\mathrm{Na}]^{+}$; found: 312.18

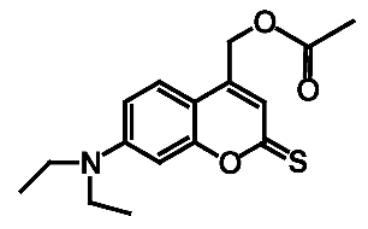

thio-DEACM-AA (6)

(7-(diethylamino)-2-thioxo-2H-chromen-4-yl)methyl acetate

Lawesson's reagent ( $229 \mathrm{mg}, 0.56 \mathrm{mmol}, 0.65$ eq.) was added to a solution of compound $\mathbf{5}$ ( $250 \mathrm{mg}$, $0.86 \mathrm{mmol}, 1$ eq.) and refluxed in dry toluene $(30 \mathrm{~mL})$ under inert gas and dark for $6 \mathrm{~h}$. The evaporation of the solvent and subsequently the recrystallization in methanol yielded compound $\mathbf{6}$ as yellow solid.

Reaction yield: $37.2 \mathrm{mg}(0.12 \mathrm{mmol}, 53.6 \%)$

Rf: 0.8 (DCM)

${ }^{1} \mathbf{H}$ NMR (DMSO-d $\left.6,250 \mathrm{MHz}\right): \delta=7.58\left(\mathrm{~d},{ }^{3} J(\mathrm{H}, \mathrm{H})=8.9 \mathrm{~Hz}, 1 \mathrm{H}\right), 6.80-6.90(\mathrm{~m}, 2 \mathrm{H})$, $6.73\left(\mathrm{~d},{ }^{4} J(\mathrm{H}, \mathrm{H})=2.4 \mathrm{~Hz}, 1 \mathrm{H}\right), 5.27(\mathrm{~s} .2 \mathrm{H}), 3.48\left(\mathrm{q},{ }^{3} J(\mathrm{H}, \mathrm{H})=7.3 \mathrm{~Hz}, 4 \mathrm{H}\right), 2.16(\mathrm{~s}, 3 \mathrm{H}$, $\left.\mathrm{CH}_{3}\right), 1.14\left(\mathrm{t},{ }^{3} \mathrm{~J}(\mathrm{H}, \mathrm{H})=6.9 \mathrm{~Hz}, 6 \mathrm{H}\right) \mathrm{ppm}$.

MS (ESI): $\mathrm{m} / \mathrm{z}$ calcd for $\mathrm{C}_{16} \mathrm{H}_{19} \mathrm{NO}_{3} \mathrm{~S}: 328.10[M+\mathrm{Na}]^{+}$; found: 328.15 


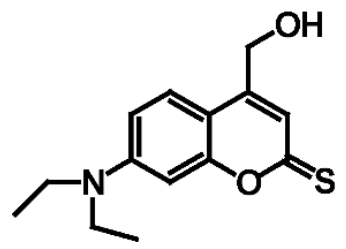

thio-DEACM-OH (7)

7-(diethylamino)-4-(hydroxymethyl)-2H-chromene-2-thione.

Derivative 6 (100 mg, 0.32, 1eq.) was solved in $20 \mathrm{~mL} 1.2 \mathrm{M} \mathrm{HCl}$ and heated for $5 \mathrm{~h}$ at $60^{\circ} \mathrm{C}$. Water was removed under HV and a column chromatography on silica gel with DCM/acetone 95:5 was performed to yield product 7 as brown solid.

Reaction yield: $19.22 \mathrm{mg}(0.07 \mathrm{mmol}, 79.4 \%)$

Rf: 0.45 (DCM/Acetone, 95:5)

${ }^{1}$ H NMR: (MeOD, $\left.500 \mathrm{MHz}\right): \delta=7.42\left(\mathrm{~d},{ }^{3} J(\mathrm{H}, \mathrm{H})=8.9 \mathrm{~Hz}, 1 \mathrm{H}\right), 7.01(\mathrm{~s}, 1 \mathrm{H}) 6.63\left(\mathrm{dd},{ }^{4} J(\mathrm{H}, \mathrm{H})\right.$ $\left.=2.6 \mathrm{~Hz},{ }^{3} J(\mathrm{H}, \mathrm{H})=9.0 \mathrm{~Hz}, 1 \mathrm{H}\right), 6.55\left(\mathrm{~d},{ }^{4} J(\mathrm{H}, \mathrm{H})=1.2 \mathrm{~Hz}, 1 \mathrm{H}\right), 4.62\left(\mathrm{~d},{ }^{4} J(\mathrm{H}, \mathrm{H})=1.3 \mathrm{~Hz}, 2\right.$ $\mathrm{H}), 3.42\left(\mathrm{q},{ }^{3} J(\mathrm{H}, \mathrm{H})=7.2 \mathrm{~Hz}, 4 \mathrm{H}\right), 1.21\left(\mathrm{t},{ }^{3} J(\mathrm{H}, \mathrm{H})=7.2 \mathrm{~Hz}, 6 \mathrm{H}\right) \mathrm{ppm}$.

${ }^{13}$ C NMR: (MeOD-d 4 , $\left.125.7 \mathrm{MHz}\right): \delta=197.8,158.8,151.2,149.0,124.7,118.7,110.4,108.2$, 96.2, 59.1, 44.3, $10.5 \mathrm{ppm}$.

MS (ESI): m/z calcd for $\mathrm{C}_{14} \mathrm{H}_{17} \mathrm{NO}_{2} \mathrm{~S}: 262.1[M-\mathrm{H}]^{-}$; found: 262.09 .

Synthesis of nitro-thio-DEACM-puromycin (I)

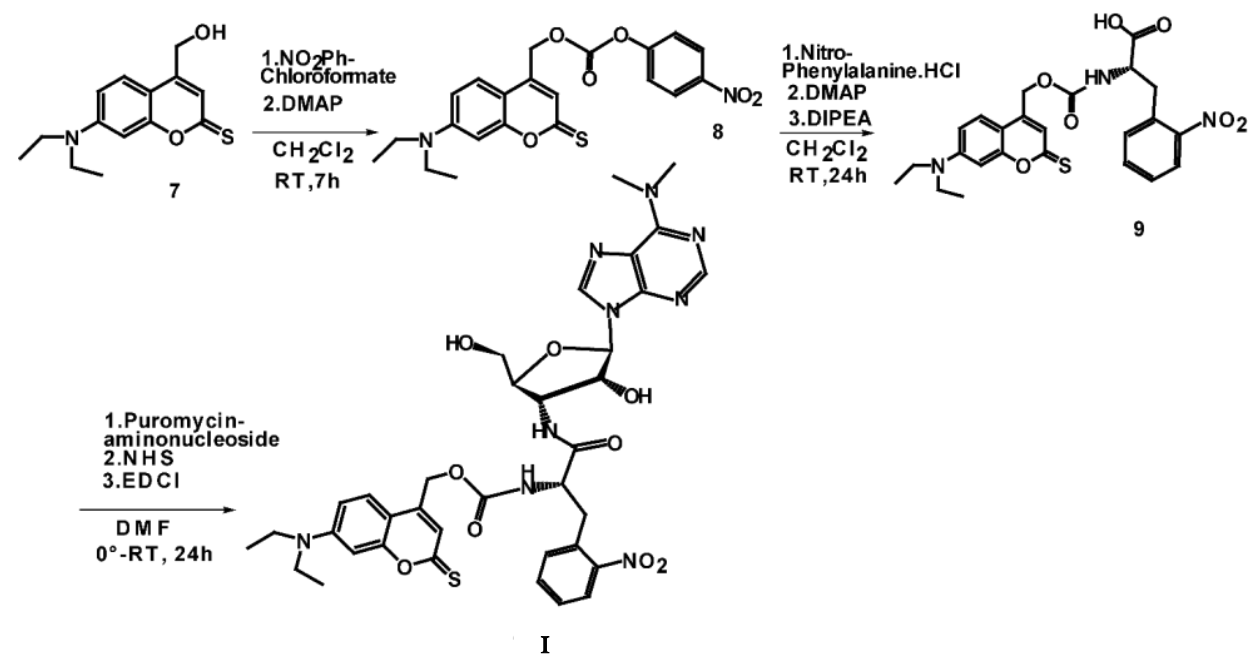




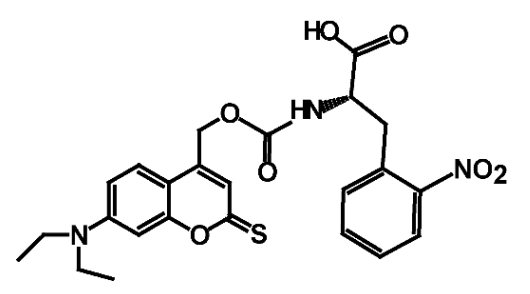

\section{$o$-nitro-Phe-Ala- $t c$ DEACM (9)}

(S)-2-(((7-(diethylamino)-2-thioxo-2H-chromen-4-yl)methoxy)carbonyl)-3-(2- nitrophenyl)propanoic acid.

\section{Step 1. Carbonate intermediate (8)}

To a solution of thio-DEACM 7 (50 mg, $0.19 \mathrm{mmol}, 1$ eq.) in $2 \mathrm{~mL}$ dried dichloromethane, DIPEA (0.6 mL, $3.8 \mathrm{mmol}, 20$ eq.), DMAP ( $51 \mathrm{mg}, 0.39 \mathrm{mmol}, 2.2$ eq.) and 4-nitrophenylchloroformate ( $85 \mathrm{mg}$, 0.39 mmol, 2.2 eq.) were added and stirred under $\mathrm{N}_{2}$ at room temperature and minimizing of light exposure for $6 \mathrm{~h}$. the crude brown mixture was used in the next step for synthesizing caged nitrophenylalanine 9 without further purification.

\section{Step 2. Carbamate product (9)}

To the reaction mixture with intermediate 8, 2-Nitro-L-phenylalanine ( $40 \mathrm{mg}, 0.19 \mathrm{mmol}, 1$ eq.), DMAP ( $51 \mathrm{mg}, 0.39 \mathrm{mmol}, 2.2 \mathrm{eq}$.) were added and stirred under same conditions for a further $16 \mathrm{~h}$. The brown solution was diluted with DCM and washed with conc. $\mathrm{NaHCO}_{3}$ solution, brine and $\mathrm{H}_{2} \mathrm{O}$. After drying with $\mathrm{MgSO}_{4}$ the solvent was removed in vacuo. To purify the desired product $\mathbf{9}$, silica gel chromatography (DCM/MeOH, gradient, 19:1, 9:1, 1:1), subsequently reversed-phase HPLC $\left(\mathrm{H}_{2} \mathrm{O} / \mathrm{ACN}\right)$ and lyophilisation afforded the title compound 9 as yellow needles.

Reaction yield: $40 \mathrm{mg}(0.08 \mathrm{mmol}, 43 \%, 2$ steps $)$

$\mathbf{R}_{\mathbf{f}}: 0.37(\mathrm{DCM} / \mathrm{MeOH}, 1: 1)$

${ }^{1}$ H NMR: (DMSO-d $\left.6,500 \mathrm{MHz}\right): \delta=7.49\left(\mathrm{~d},{ }^{3} J(\mathrm{H}, \mathrm{H})=8.4 \mathrm{~Hz}, 1 \mathrm{H}\right), 7.62-7.68(\mathrm{~m}, 1 \mathrm{H}), 7.45-$ $7.54(\mathrm{~m}, 5 \mathrm{H}), 6.81(\mathrm{~s}, 2 \mathrm{H}), 6.73\left(\mathrm{~d},{ }^{4} J(\mathrm{H}, \mathrm{H})=2.4 \mathrm{~Hz}, 1 \mathrm{H}\right), 5.14(\mathrm{~s}, 2 \mathrm{H}), 3.84\left(\mathrm{q},{ }^{3} J(\mathrm{H}, \mathrm{H})=\right.$ $7.2 \mathrm{~Hz}, 4 \mathrm{H}), 3.1\left(\mathrm{dd},,{ }^{3} J(\mathrm{H}, \mathrm{H})=12.1 \mathrm{~Hz},{ }^{4} J(\mathrm{H}, \mathrm{H})=3.3 \mathrm{~Hz}, 1 \mathrm{H}\right), 1.14\left(\mathrm{t},{ }^{3} J(\mathrm{H}, \mathrm{H})=6.9 \mathrm{~Hz}, 6\right.$ H) ppm.

HSQC (DMSO, 500/125 MHz): $\delta=18.4,28.2,54.0,81.6,98.5,109.4,109.6,110.4,126.5$, $151.7,154.0,155.3,161.0,169.4 \mathrm{ppm}$.

MS (ESI): $\mathrm{m} / \mathrm{z}$ calcd for $\mathrm{C}_{24} \mathrm{H}_{25} \mathrm{~N}_{3} \mathrm{O}_{7} \mathrm{~S}: 500.14[M+\mathrm{H}]^{+}$; found: 500.14 


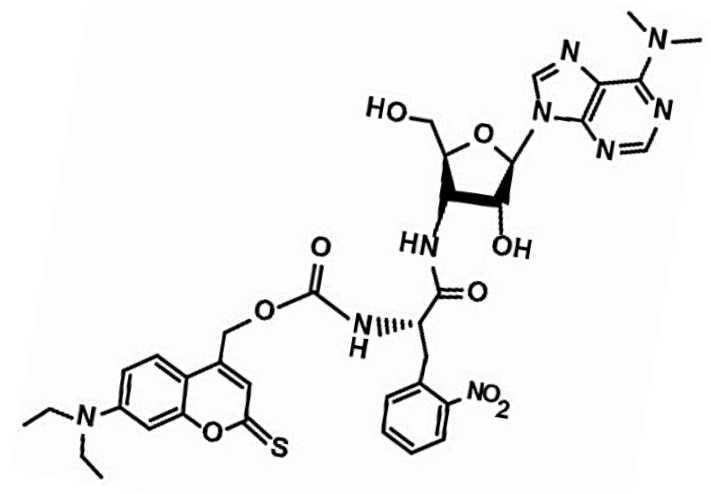

thio-DEACM-nitro-puromycin (I)

(7-(diethylamino)-2-thioxo-2H-chromen-4-yl)methyl (S)-1-((2S,3S,4R,5R)-5-(6-(dimethylamino)-9H-purin-9-yl)-4-hydroxy2-(hydroxymethyl)-tetrahydrofuran-3-ylamino)-3-(2-nitrophenyl)-1-oxopropan-2-ylcarbamate.

A mixture of synthesized compound 9 ( $40 \mathrm{mg}, 0.08 \mathrm{mmol}, 1$ eq.), aminopuromycin nucleoside (24 $\mathrm{mg}, 0.08 \mathrm{mmol}$, 1eq.) and NHS (10 mg, $0.08 \mathrm{mmol}$, 1eq.) in $2 \mathrm{~mL} \mathrm{DMF}$ was cooled to $0^{\circ} \mathrm{C}$. EDCI (16 $\mathrm{mg}, 0.08 \mathrm{mmol}$, 1eq.) was added and the solution was stirred under $\mathrm{N}_{2}$ in dark for $16 \mathrm{~h}$. The obtained brown mixture was quenched with $4 \mathrm{~mL} \mathrm{H}_{2} \mathrm{O} / \mathrm{ACN}$ 1:1. Precipitated yellow solid was collected and washed with $\mathrm{H}_{2} \mathrm{O}$ and purified with reversed-phase $\mathrm{HPLC}\left(\mathrm{H}_{2} \mathrm{O} / \mathrm{ACN}\right)$.

Reaction yield: $18 \mathrm{mg}(0.02 \mathrm{mmol}, 29 \%)$

Rf: $0.15(\mathrm{DCM} / \mathrm{MeOH}, 1: 1)$

${ }^{1}$ H NMR (DMSO, $\left.500 \mathrm{MHz}\right): \delta=8.24(\mathrm{~s}, 1 \mathrm{H}), 8.24(\mathrm{~s} 1 \mathrm{H}), 8.10\left(\mathrm{~d},{ }^{3} J(\mathrm{H}, \mathrm{H})=7.9 \mathrm{~Hz}, 1 \mathrm{H}\right), 7.99(\mathrm{~d}$, $\left.{ }^{3} J(\mathrm{H}, \mathrm{H})=8.2 \mathrm{~Hz}, 1 \mathrm{H}\right), 7.92\left(\mathrm{td},{ }^{3} J(\mathrm{H}, \mathrm{H})=7.7 \mathrm{~Hz},{ }^{4} J(\mathrm{H}, \mathrm{H})=0.9 \mathrm{~Hz}, 1 \mathrm{H}\right) 7.45-7.55(\mathrm{~m}, 3 \mathrm{H}), 6.86(\mathrm{~s}$, $1 \mathrm{H}), 6.81\left(\mathrm{dd},{ }^{3} J(\mathrm{H}, \mathrm{H})=9.4 \mathrm{~Hz},{ }^{4} J(\mathrm{H}, \mathrm{H})=2.8 \mathrm{~Hz}, 1 \mathrm{H}\right), 6.73(\mathrm{~s}, 1 \mathrm{H}), 5.98\left(\mathrm{~d},{ }^{3} J(\mathrm{H}, \mathrm{H})=3.2 \mathrm{~Hz}, 1 \mathrm{H}\right)$, 5.10-5.24 (m, $2 \mathrm{H}), 4.43-4.60(\mathrm{~m}, 3 \mathrm{H}), 3.89-3.93(\mathrm{~m}, 1 \mathrm{H}), 3.65\left(\mathrm{dd},,{ }^{3} J(\mathrm{H}, \mathrm{H})=12.5 \mathrm{~Hz},{ }^{4} J(\mathrm{H}, \mathrm{H})=2.2\right.$ $\mathrm{Hz}, 1 \mathrm{H}), 3.84\left(\mathrm{q},{ }^{3} J(\mathrm{H}, \mathrm{H})=7.2 \mathrm{~Hz}, 4 \mathrm{H}\right), 1.13\left(\mathrm{t},{ }^{3} J(\mathrm{H}, \mathrm{H})=7.5 \mathrm{~Hz}, 6 \mathrm{H}\right) \mathrm{ppm}$.

HSQC (DMSO, 500/125 MHz): $\delta=150.8,138.7,133.4,133.2,128.3,126.0,124.9,119.1$, $111.5,96.9,89.4,83.4,72.7,61.0,60.2,55.2,51.1,44.6$ ppm.

MS (ESI): m/z calcd for $\mathrm{C}_{36} \mathrm{H}_{41} \mathrm{~N}_{9} \mathrm{O}_{9} \mathrm{~S}: 776.27[M+\mathrm{H}]^{+}$; found: 776.21 


\section{Synthesis of $\boldsymbol{o}$-nitro-puromycin (II)}
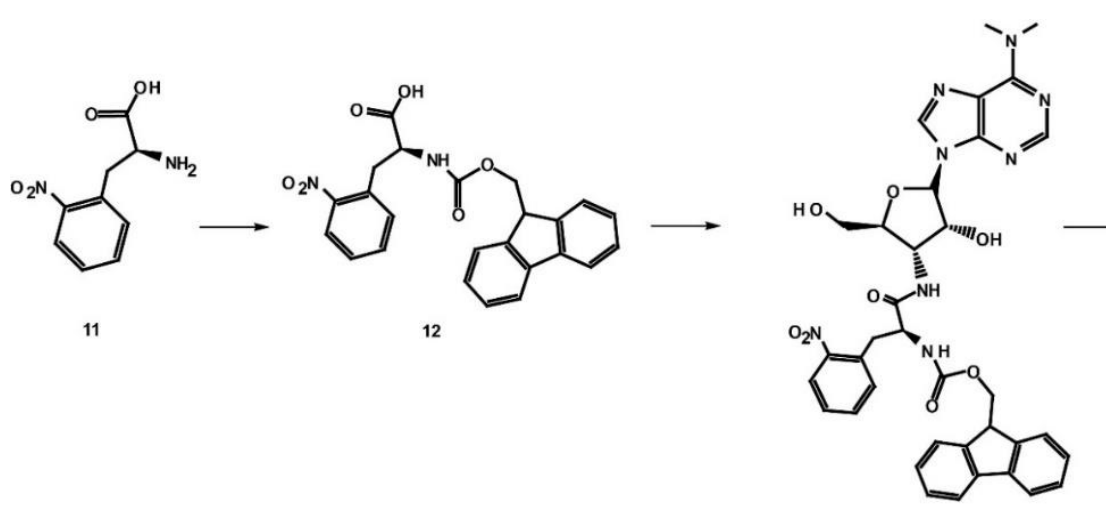

13
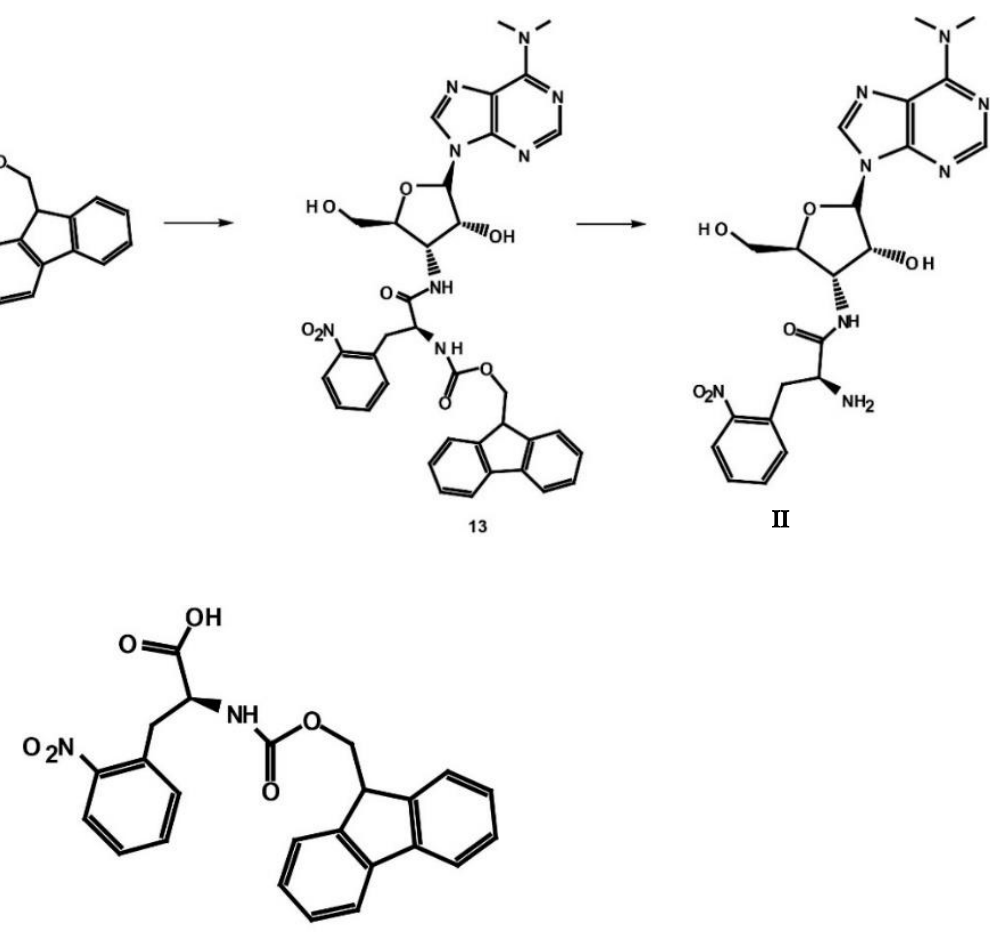

Fmoc-L-2.nitrophenylalanine (12)

(S)-2-(((9H-fluoren-9-yl)methoxy)carbonyl)-3-(2-nitrophenyl)propanoic acid

9-fluorenylmethylchloroformate ( $150 \mathrm{mg}, 0.57 \mathrm{mmol}, 1.1$ eq.) dissolved in $2 \mathrm{~mL}$ dioxane was added dropwise to a stirred solution containing 2-Nitro-L-phenylalanine (225 mg, $0.52 \mathrm{mmol}, 1 \mathrm{eq}$.), 10\% aqueous $\mathrm{Na}_{2} \mathrm{CO}_{3}(1.5 \mathrm{~mL})$ that was cooled in an ice bad. After stirring at $0^{\circ} \mathrm{C}$ for $1 \mathrm{~h}$, the reaction mixture was stirring for $3 \mathrm{~h}$ at ambient temperature. Than was quenched with $50 \mathrm{~mL} \mathrm{H}_{2} \mathrm{O}$ and extracted with $\mathrm{Et}_{2} \mathrm{O}(3 \times 50 \mathrm{~mL})$. Aqueous layers were acidified with conc. $\mathrm{HCl}$ and extracted with EtOAc. subsequently, the organic layers were dried over $\mathrm{MgSO}_{4}$ and purified with help of silica gel chromatography (step gradient: $0-1 \% \mathrm{AcOH}$ in $1: 1$ hexanes/Et ${ }_{2} \mathrm{O}$ ) and finally recrystallized from acetonitrile to yield the protected amino acid as yellow crystalline solid.

Reaction yield: $210 \mathrm{mg}(0.49 \mathrm{mmol}, 93 \%)$

Rf: $0.20\left(\mathrm{Hex} / \mathrm{Et}_{2} \mathrm{O} / \mathrm{AcOH}, 1: 1: 1 \%\right)$

${ }^{1}$ H NMR: (DMSO-d $\left.6,500 \mathrm{MHz}\right): \delta=12.9(\mathrm{br}, 1 \mathrm{H}) 7.9\left(\mathrm{dd},{ }^{3} \mathrm{~J}(\mathrm{H}, \mathrm{H})=8.5 \mathrm{~Hz},{ }^{4} \mathrm{~J}(\mathrm{H}, \mathrm{H})=0.9 \mathrm{~Hz}\right.$, $1 \mathrm{H}), 7.9\left(\mathrm{~d}, 6.63,{ }^{3} J(\mathrm{H}, \mathrm{H})=7.6 \mathrm{~Hz}, 2 \mathrm{H}\right), 7.8\left(\mathrm{~d},{ }^{3} J(\mathrm{H}, \mathrm{H})=7.6 \mathrm{~Hz}, 1 \mathrm{H}\right), 7.59-7.64(\mathrm{~m}, 3 \mathrm{H})$, 7.47-7.53 (m, 2H), $7.42\left(\mathrm{td},{ }^{3} J(\mathrm{H}, \mathrm{H})=7.5 \mathrm{~Hz},{ }^{4} J(\mathrm{H}, \mathrm{H})=2.2 \mathrm{~Hz}, 2 \mathrm{H}\right), 7.23-7.35(\mathrm{~m}, 2 \mathrm{H}), 4.36-$ $4.4(\mathrm{~m}, 1 \mathrm{H}), 4.13-4,23(\mathrm{~m}, 3 \mathrm{H}), 3.5\left(\mathrm{dd},{ }^{3} J(\mathrm{H}, \mathrm{H})=13.8 \mathrm{~Hz}, 1 \mathrm{H}\right), 3.1\left(\mathrm{q},{ }^{3} J(\mathrm{H}, \mathrm{H})=12.5 \mathrm{~Hz}, 1 \mathrm{H}\right)$. 
${ }^{13}$ C NMR (DMSO-d $\left.6,125 \mathrm{MHz}\right): \delta=173.3,156.4,149.7,144.2,141.2,133.5,132.8,128.6$, $128.1,127.5,125.7,125.0,120.6,66.1,54.3,47.0,34.1 \mathrm{ppm}$.

MS (ESI): $\mathrm{m} / \mathrm{z}$ calcd for $\mathrm{C}_{24} \mathrm{H}_{20} \mathrm{~N}_{2} \mathrm{O}_{6}: 433.13\left[M+\mathrm{H}^{+}\right.$; found: 433.12

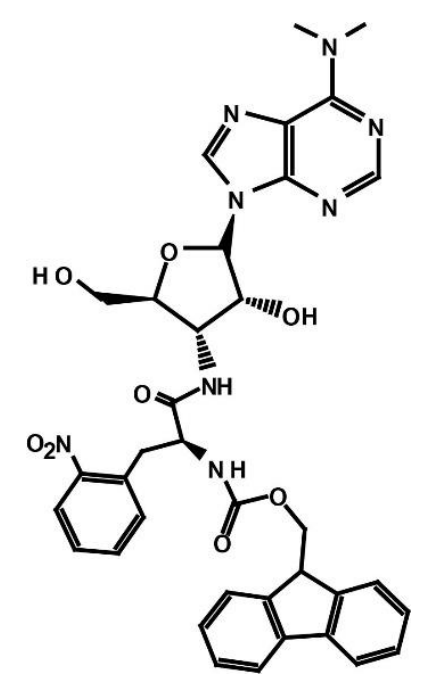

Fmoc-nitro-puromycin

(9H-fluoren-9-yl)methyl (S)-1-((2S,3S,4R,5S)-5-(6-(dimethylamino)-9H-purin-9-yl)-4-hydroxy-2- ～(hydroxymethyl)tetrahydrofuran-3-ylamino)-3-(2-nitrophenyl)-1-oxopropan-2-ylcarbamate

A mixture of synthesized compound 12 (74 mg, $0.17 \mathrm{mmol}, 1$ eq.), aminopuromycin nucleoside (50 $\mathrm{mg}, 0.17 \mathrm{mmol}$, 1eq.) and NHS (21 mg, $0.08 \mathrm{mmol}$, 1eq.) in $2 \mathrm{~mL} \mathrm{DMF}$ was cooled to $0^{\circ} \mathrm{C}$. EDCI (34 $\mathrm{mg}, 0.17 \mathrm{mmol}$, 1eq.) was added and the solution was stirred under $\mathrm{N}_{2}$ in dark for $16 \mathrm{~h}$. The obtained brown mixture was quenched with cold $\mathrm{H}_{2} \mathrm{O}$ and extracted with EtOAc than Precipitated from $4 \mathrm{~mL}$ $\mathrm{H}_{2} \mathrm{O} / \mathrm{ACN}$ 1:1 to give product $\mathbf{1 3}$ as white solid that was collected and washed with $\mathrm{H}_{2} \mathrm{O}$.

Reaction yield: $60 \mathrm{mg}(0.08 \mathrm{mmol}, 47 \%)$

Rf: $0.70(\mathrm{DCM} / \mathrm{MeOH}, 9: 1)$

${ }^{1}$ H NMR: (DMSO-d $\left.6,600 \mathrm{MHz}\right): \delta=8.49$ (s, 1H), 8.29 (s, 1H), 8.1(dd, ${ }^{3} J(\mathrm{H}, \mathrm{H})=8.1 \mathrm{~Hz}, 2$ $\mathrm{H}), 7.94\left(\mathrm{~d},{ }^{3} J(\mathrm{H}, \mathrm{H})=7.7 \mathrm{~Hz}, 2 \mathrm{H}\right), 7.77\left(\mathrm{~d},{ }^{3} J(\mathrm{H}, \mathrm{H})=8.7 \mathrm{~Hz}, 1 \mathrm{H}\right), 7.71\left(\mathrm{q},{ }^{3} J(\mathrm{H}, \mathrm{H})=7.9 \mathrm{~Hz}\right.$, $\left.{ }^{4} J(\mathrm{H}, \mathrm{H})=3.9 \mathrm{~Hz}, 2 \mathrm{H}\right), 7.63-7.66(\mathrm{~m}, 1 \mathrm{H}), 7.59\left(\mathrm{~d},{ }^{3} J(\mathrm{H}, \mathrm{H})=7.3 \mathrm{~Hz}, 1 \mathrm{H}\right), 7.52-7.55(\mathrm{~m}, 1 \mathrm{H})$, 7.44-7.51 (m, $2 \mathrm{H}), 7.35-7.40(\mathrm{~m}, 2 \mathrm{H}), 6.1\left(\mathrm{~d},{ }^{3} J(\mathrm{H}, \mathrm{H})=4.7 \mathrm{~Hz}, 1 \mathrm{H}\right), 6.03\left(\left(\mathrm{~d},{ }^{3} J(\mathrm{H}, \mathrm{H})=3.3\right.\right.$ $\mathrm{Hz}, 1 \mathrm{H}), 5.24\left(\mathrm{t},{ }^{3} J(\mathrm{H}, \mathrm{H})=5.4 \mathrm{~Hz}, 1 \mathrm{H}\right), 4.50-4.64(\mathrm{~m}, 3 \mathrm{H}), 4.17-4.28(\mathrm{~m}, 3 \mathrm{H}), 3.97(\mathrm{~m}, 1 \mathrm{H})$, 
$3.72(\mathrm{~m}, 2 \mathrm{H}), 3.49-3.54(\mathrm{~m}, 4 \mathrm{H}), 3.2\left(\mathrm{q},{ }^{3} J(\mathrm{H}, \mathrm{H})=12.5 \mathrm{~Hz}, 1 \mathrm{H}\right) . \quad$ MS (ESI): $\mathrm{m} / \mathrm{z}$ calcd for $\mathrm{C}_{36} \mathrm{H}_{36} \mathrm{~N}_{8} \mathrm{O}_{8}: 709.27[M+\mathrm{H}]^{+}$; found: 710.61

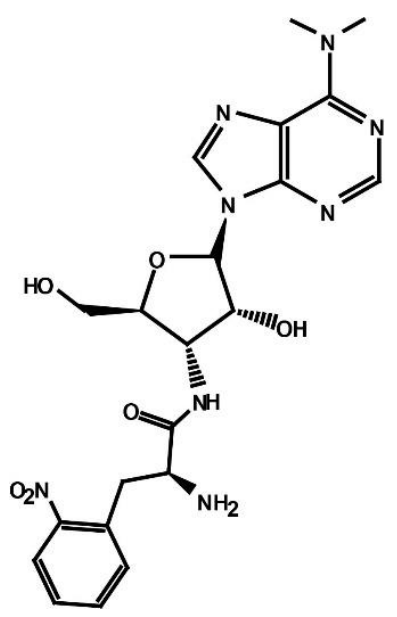

\section{$o$-nitro-puromycin (II)}

(S)-2-amino-N-((2S,3S,4R,5S)-5-(6-(dimethylamino)-9H-purin-9-yl)-4-hydroxy-2-(hydroxymethyl)-tetrahydrofuran-3-yl)3-(2-nitrophenyl) propanamide

Compound 13 (50 mg, $0.07 \mathrm{mmol}, 1$ eq.) was dissolved in a mixture of DMF and morpholine (1.5 $\mathrm{mL1}: 1)$ and stirred at room temperature under inert gas in dark for $2 \mathrm{~h}$. FMOC-morpholine was removed by precipitation with $\mathrm{H}_{2} \mathrm{O}$. The suspension was centrifuged and filtered out.

Reaction yield: $28 \mathrm{mg}(0.06 \mathrm{mmol}, 82 \%)$

Rf: $0.70(\mathrm{DCM} / \mathrm{MeOH}, 8: 2)$

${ }^{1} \mathbf{H}$ NMR: (DMSO-d $\left.6,500 \mathrm{MHz}\right): \delta=8.45 \quad(\mathrm{~s}, \quad 1 \quad \mathrm{H}), \quad 8.25 \quad(\mathrm{~s}, \quad 1 \quad \mathrm{H})$, $8.13(\mathrm{br}, 1 \mathrm{H}), 7.94\left(\mathrm{~d},{ }^{3} J(\mathrm{H}, \mathrm{H})=7.9 \mathrm{~Hz}, 1 \mathrm{H}\right), 7.65\left(\mathrm{td},{ }^{3} J(\mathrm{H}, \mathrm{H})=7.6 \mathrm{~Hz},{ }^{4} J(\mathrm{H}, \mathrm{H})=1.2 \mathrm{~Hz}\right.$, $1 \mathrm{H}), 7.55\left(\mathrm{~d},{ }^{3} J(\mathrm{H}, \mathrm{H})=7.4 \mathrm{~Hz}, 1 \mathrm{H}\right), 7.49\left(\mathrm{t},{ }^{3} J(\mathrm{H}, \mathrm{H})=7.7 \mathrm{~Hz}, 1 \mathrm{H}\right), 6.1\left(\mathrm{~d},{ }^{3} J(\mathrm{H}, \mathrm{H})=4.9\right.$ $\mathrm{Hz}, 1 \mathrm{H}), 5.9\left(\mathrm{~d},{ }^{3} J(\mathrm{H}, \mathrm{H})=2.9 \mathrm{~Hz}, 1 \mathrm{H}\right), 5.17\left(\mathrm{t},{ }^{3} J(\mathrm{H}, \mathrm{H})=5.4 \mathrm{~Hz}, 1 \mathrm{H}\right), 4.42-4.51(\mathrm{~m}, 2 \mathrm{H})$, $3.91(\mathrm{~m}, 1 \mathrm{H}), 3.67(\mathrm{~m}, 1 \mathrm{H}), 3.2\left(\mathrm{dd}, 7.49\left(\mathrm{t},{ }^{3} J(\mathrm{H}, \mathrm{H})=5.6 \mathrm{~Hz}, 1 \mathrm{H}\right), 3.0(\mathrm{~m}, 1 \mathrm{H}) \mathrm{ppm}\right.$.

MS (ESI): $\mathrm{m} / \mathrm{z}$ calcd for $\mathrm{C}_{21} \mathrm{H}_{26} \mathrm{~N}_{8} \mathrm{O}_{6}: 486.2[M+\mathrm{H}]^{+}$; found: 485.34 


\section{Synthesis of substituted 4-hydroxy-coumarylmethyl-derivatives}

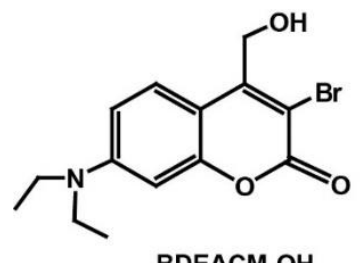

BDEACM-OH<smiles>O=c1cc(CO)c2cc(Br)c(O)cc2o1</smiles>

Bhc-OH

The synthesis of Bhc-OH was performed according to the described procedure of [R.Y.Tsien et al.] $]^{[3]}$

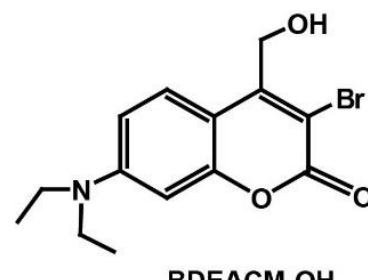

BDEACM-OH

3-bromo-7-(diethylamino)-4-(hydroxymethyl)-2H-chromen-2-one

DEACM-OH 4 (309 mg, $1.4 \mathrm{mmol}, 1$ eq.) was solved in THF (6 mL) and cooled to $0^{\circ} \mathrm{C}$. $\mathrm{N}$ bromosuccinimide ( $25 \mathrm{mg}, 1.3 \mathrm{mmol}$, and 1.1 eq.) and ammonium acetate $(9.5 \mathrm{mg}, 0.13 \mathrm{mmol}, 0.1$ eq.) were added and stirred at ambient temperature for $2 \mathrm{~h}$. the reaction was controlled with TLC. To the reaction mixture was added $\mathrm{H}_{2} \mathrm{O}$ and extracted with EtOAc. After washing with water and brine solution, the combined organic layers were dried over $\mathrm{MgSO}_{4}$. The crude product was than purified by silica chromatography (Hex/EtOAc, step gradient: 20:1, 3:1) to yield the compound as yellow crystalline solid.

Reaction yield: $210 \mathrm{mg}(0.6 \mathrm{mmol}, 43 \%)$

Rf: 0.25 (Hex/EE, 3:1)

${ }^{1}$ H NMR: $\left(\mathrm{CDCl}_{3}, 250 \mathrm{MHz}\right): \delta=7.60\left(\mathrm{~d},{ }^{3} J(\mathrm{H}, \mathrm{H})=9.2 \mathrm{~Hz}, 1 \mathrm{H}\right), 6.58\left(\mathrm{dd},{ }^{4} J(\mathrm{H}, \mathrm{H})=2.6 \mathrm{~Hz}\right.$, $\left.{ }^{3} J(\mathrm{H}, \mathrm{H})=9.1 \mathrm{~Hz}, 1 \mathrm{H}\right), 6.43\left(\mathrm{~d},{ }^{4} J(\mathrm{H}, \mathrm{H})=1.2 \mathrm{~Hz}, 1 \mathrm{H}, \mathrm{CH}\right), 4.90(\mathrm{~s}, 2 \mathrm{H}), 3.35\left(\mathrm{q},{ }^{3} J(\mathrm{H}, \mathrm{H})=\right.$ $\left.7.2 \mathrm{~Hz}, 4 \mathrm{H}, \mathrm{CH}_{2}\right), 1.14\left(\mathrm{t},{ }^{3} \mathrm{~J}(\mathrm{H}, \mathrm{H})=7.2 \mathrm{~Hz}, 6 \mathrm{H}, \mathrm{CH}_{3}\right)$.

MS (ESI): $\mathrm{m} / \mathrm{z}$ calcd for $\mathrm{C}_{14} \mathrm{H}_{17} \mathrm{BrNO}_{3}: 326.03[M+\mathrm{H}]^{+}$; found: 326.19

- $\quad$ Procedures for the synthesis of DBMAC, BMAC, DCMAC and BCMAC derivatives was performed according to Kiso et al. ${ }^{[4]}$ 


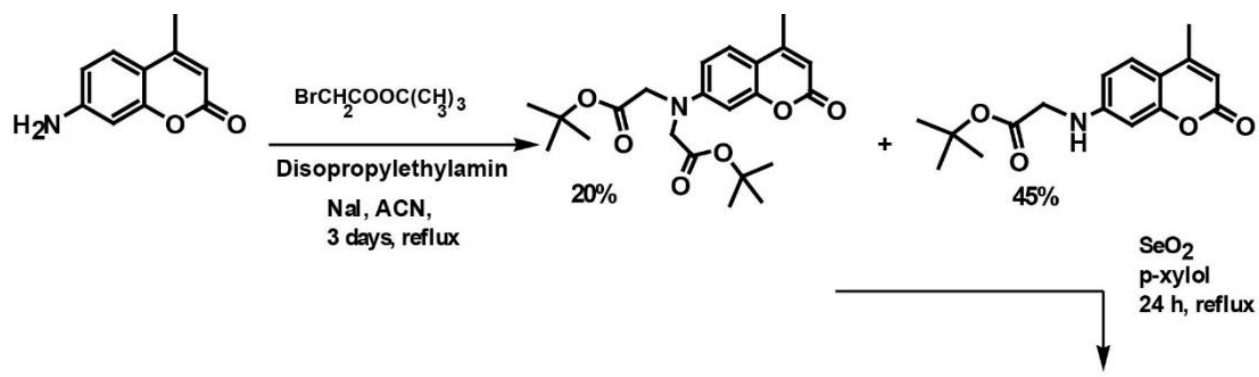

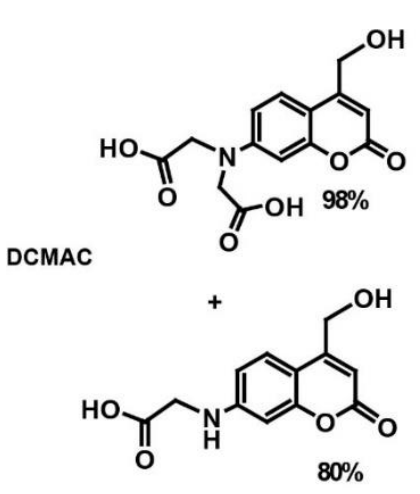

BCMAC

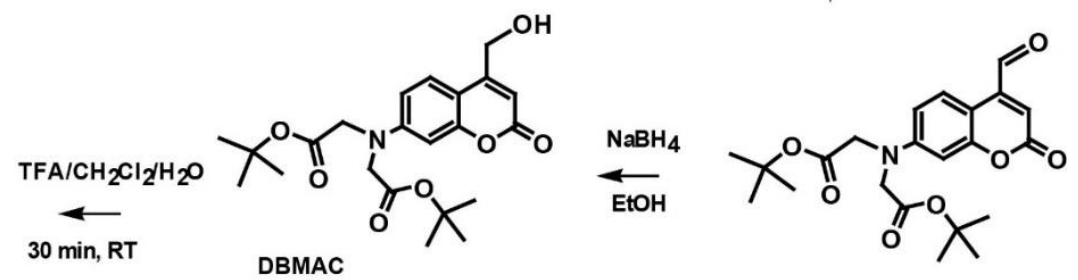

$30 \%$<smiles>CC(C)(C)OC(=O)CNc1ccc2c(CO)cc(=O)oc2c1</smiles>

BMAC

- Synthesis details of diethylamino-coumaryl-idenemalononitrilemethyl (mc-DBMAC) can be found in [jullien et al. $]^{[5]}$
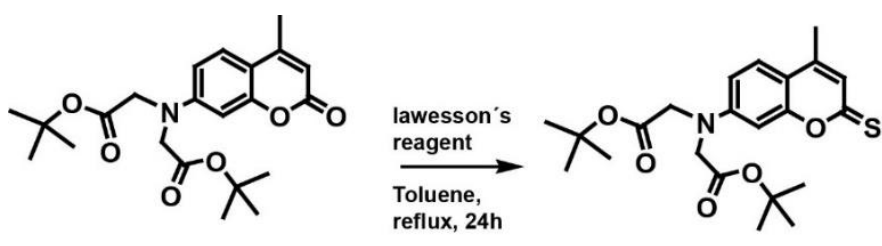

Malononitrile $\stackrel{\mathrm{PbO}, \mathrm{TEA}}{\longrightarrow}$

reflux, 24

$110^{\circ} \mathrm{C}, 24 \mathrm{~h}$

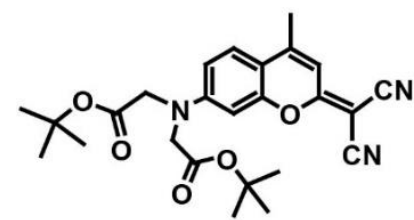<smiles></smiles>

mc-DBMAC

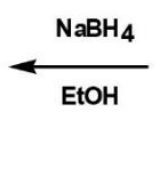<smiles></smiles> 
NMR and mass spectra
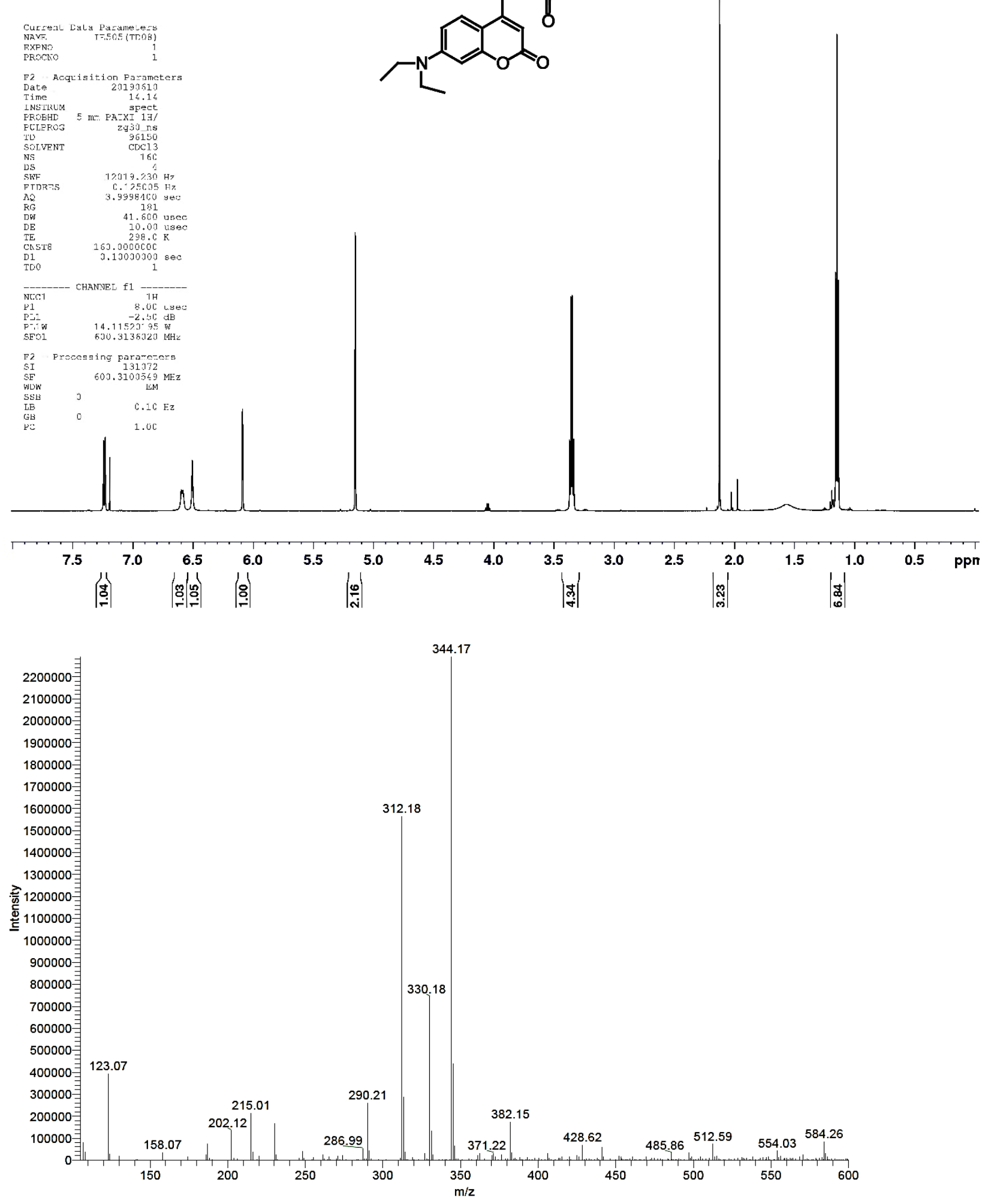

Figure S1. ${ }^{1} \mathrm{H}$ NMR- and Mass (ESI) Spectra of DEACM-AA (5) 


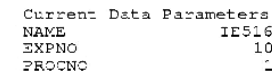

I2 - Accuistion 2azameters
Da:e_

Ta:e-
INSTRETM

INSTRJM
PROBEP
PLIPRCG

TD

NS
SW
SWA

$\mathrm{T}=\mathrm{D}$
$\mathrm{AQ}$
$\mathrm{K} G$
$\mathrm{DW}$
$\mathrm{DE}$

S
E
E
E

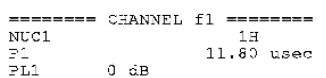

SFO1 $250.1315010 \mathrm{MH}$
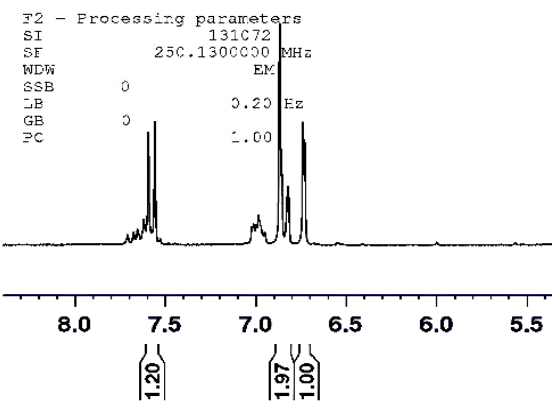
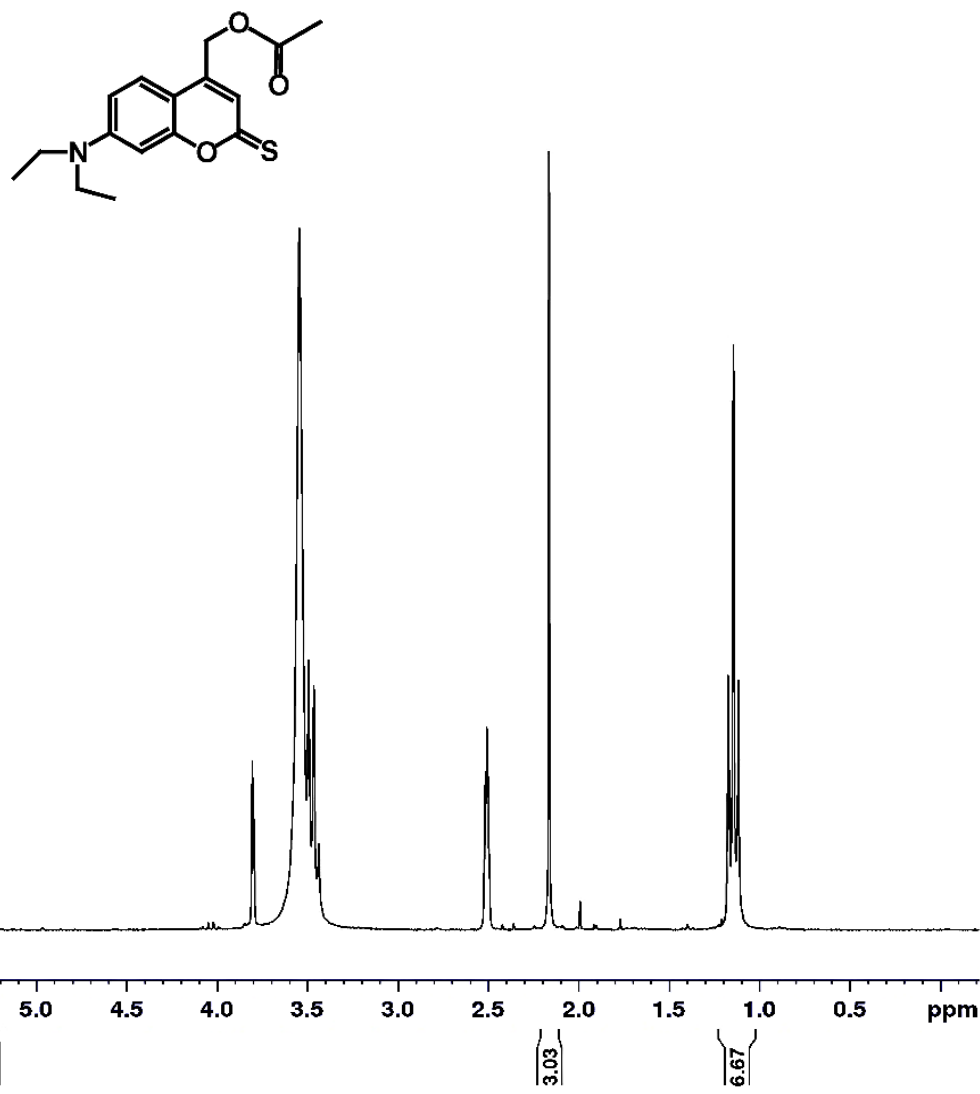

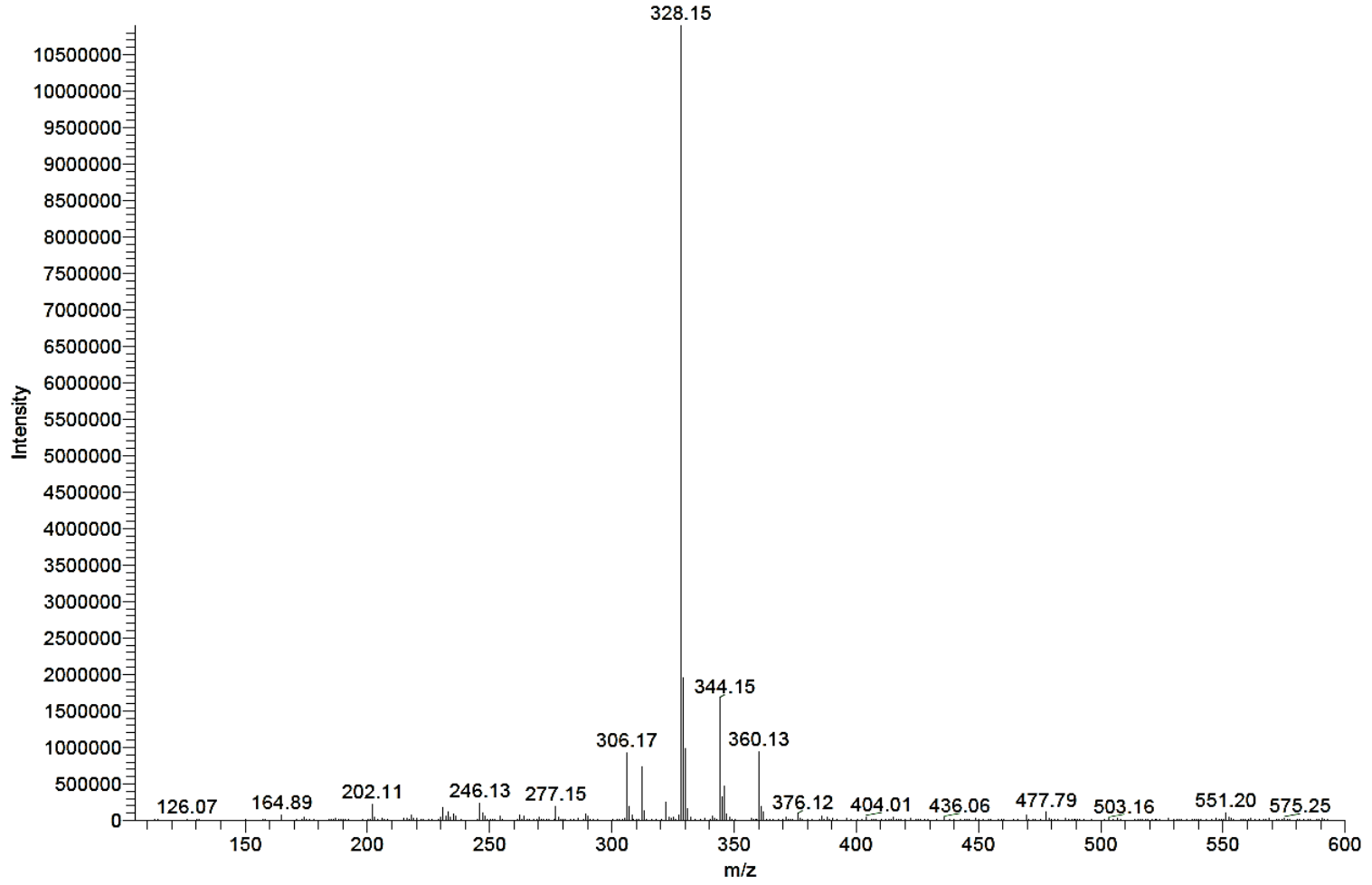

Figure S2. ${ }^{1} \mathrm{H}$ NMR- and Mass (ESI) Spectra of thio-DEACM-AA (6)

S13 

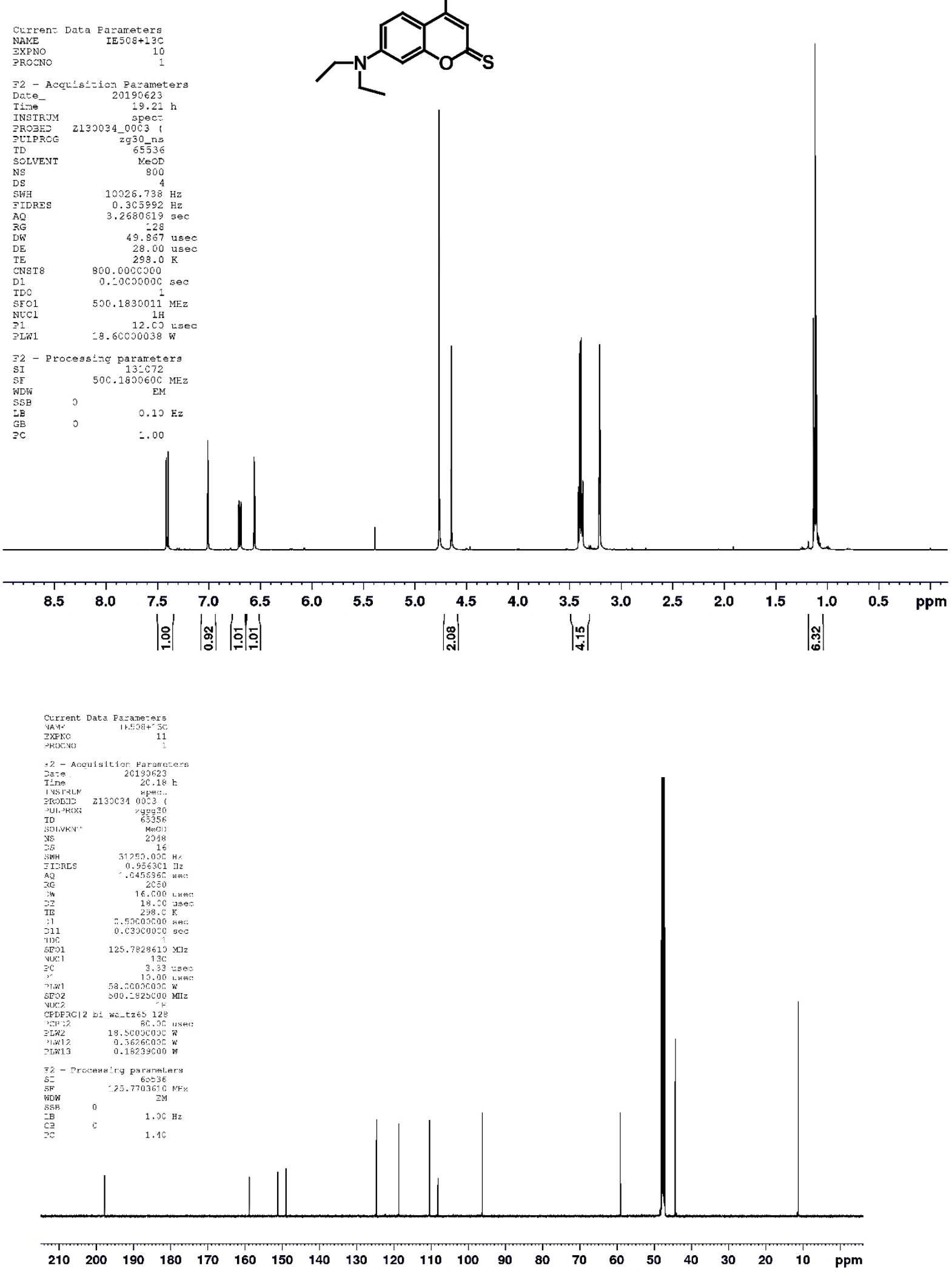

S14 


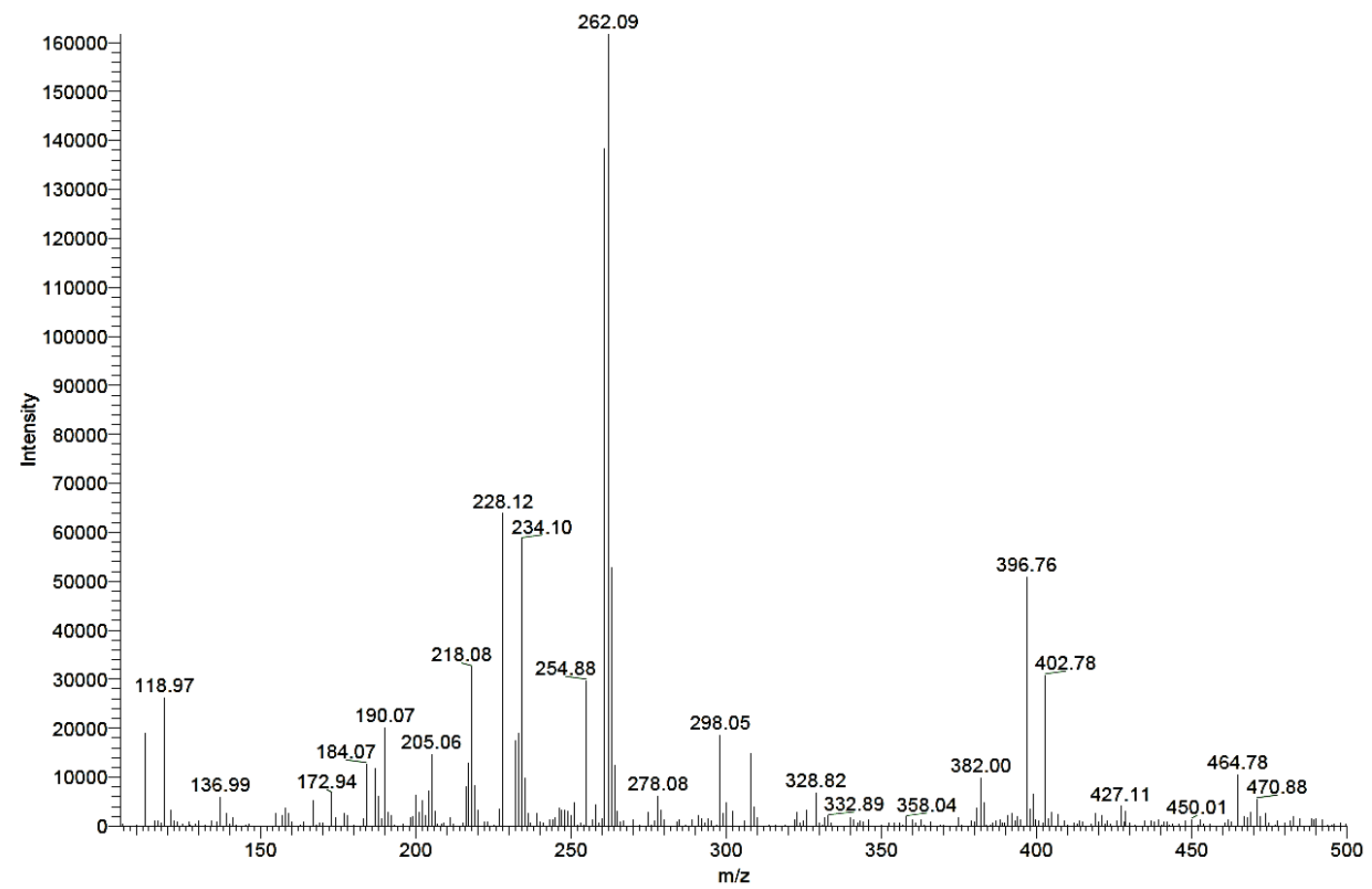

Figure S3. ${ }^{1} \mathrm{H}$ NMR-, ${ }^{13} \mathrm{C}$ NMR- and Mass (ESI) Spectra of DEACM-(7)

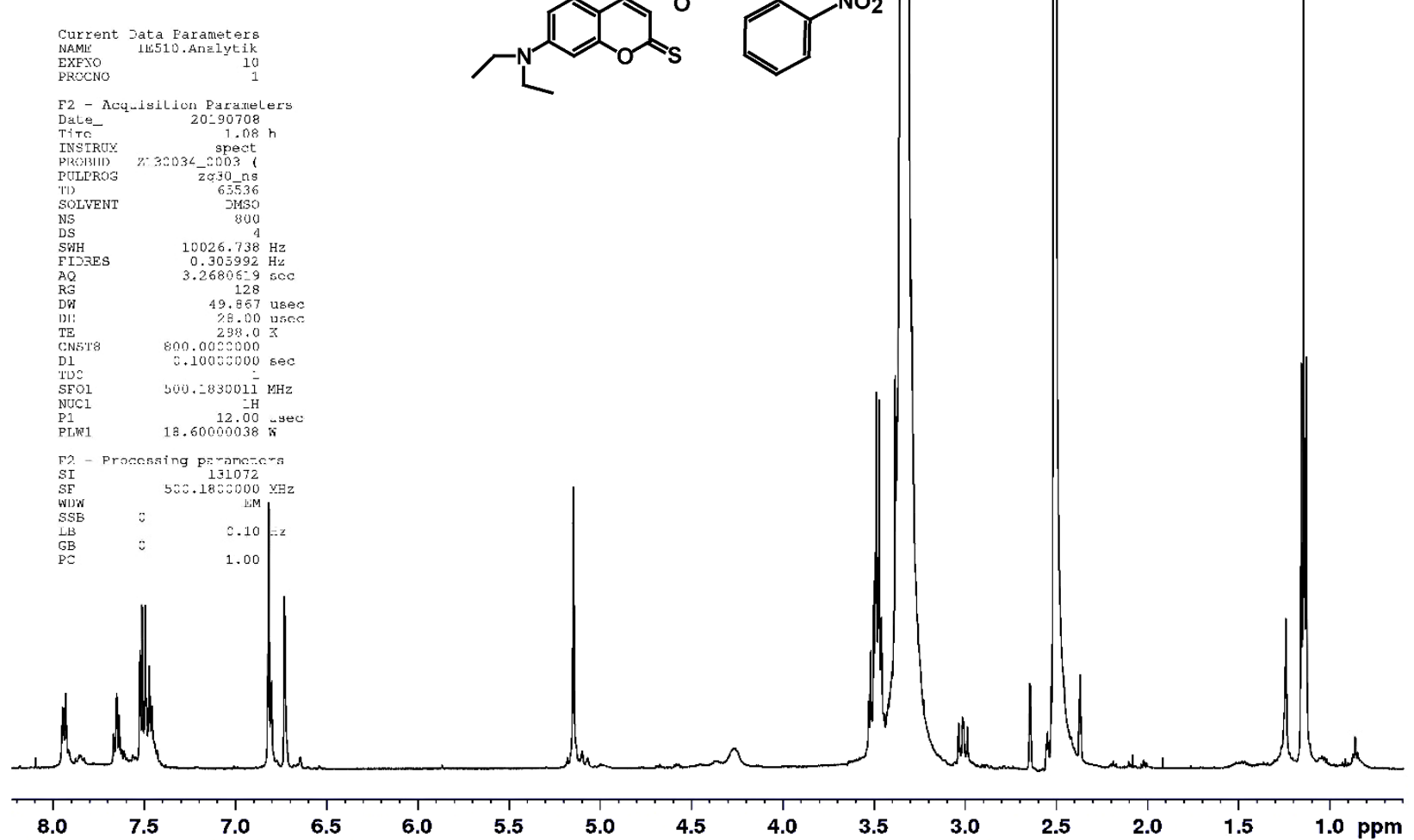



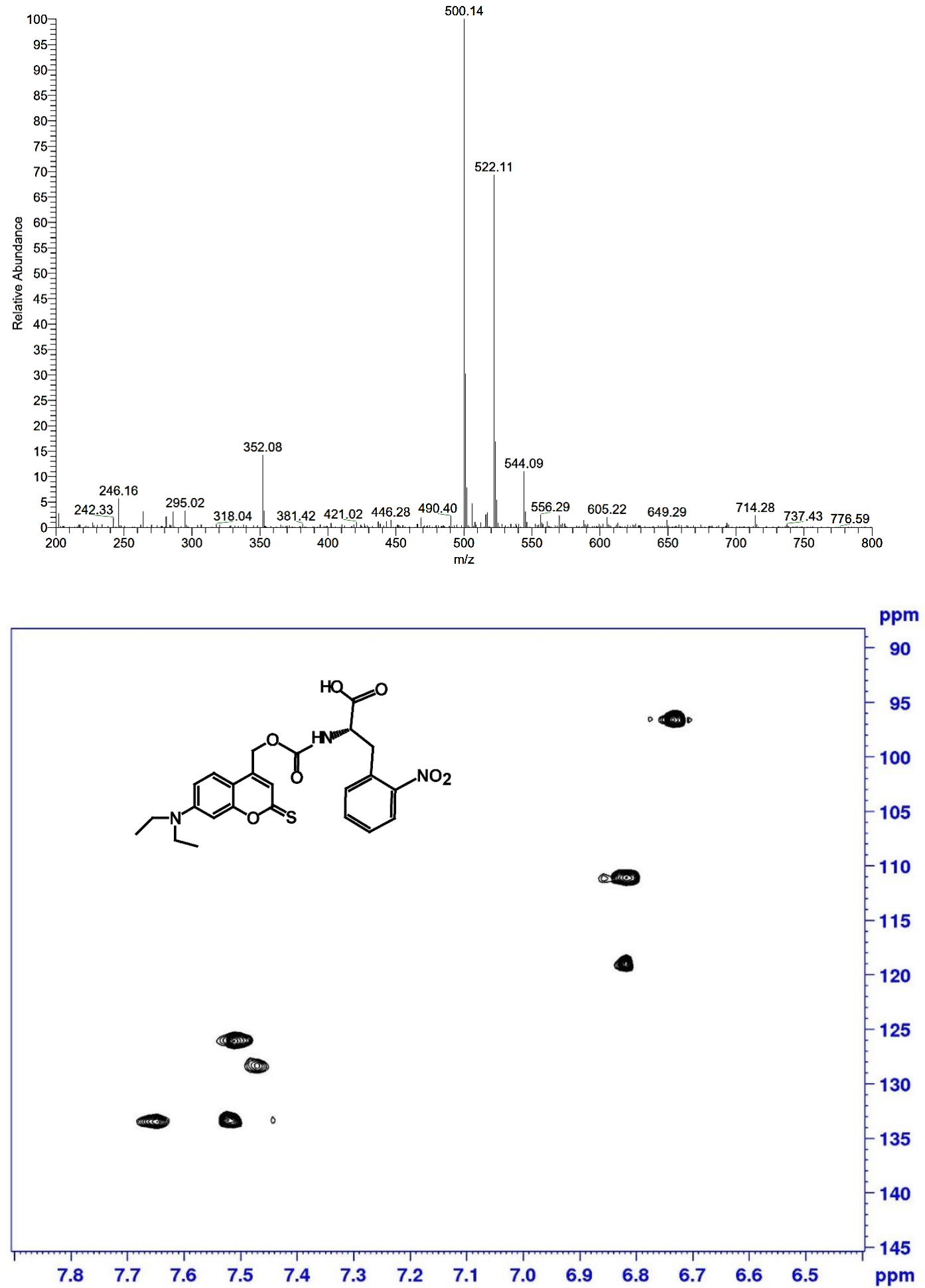

Figure S4. ${ }^{1} \mathrm{H}$ NMR-,${ }^{13} \mathrm{C}-{ }^{1} \mathrm{H}-\mathrm{HSQC}-,{ }^{1} \mathrm{H}-\mathrm{COSY}-$ and Mass (ESI) Spectra of thio-DEACMnitro- phenylalanine carbamate 9. 


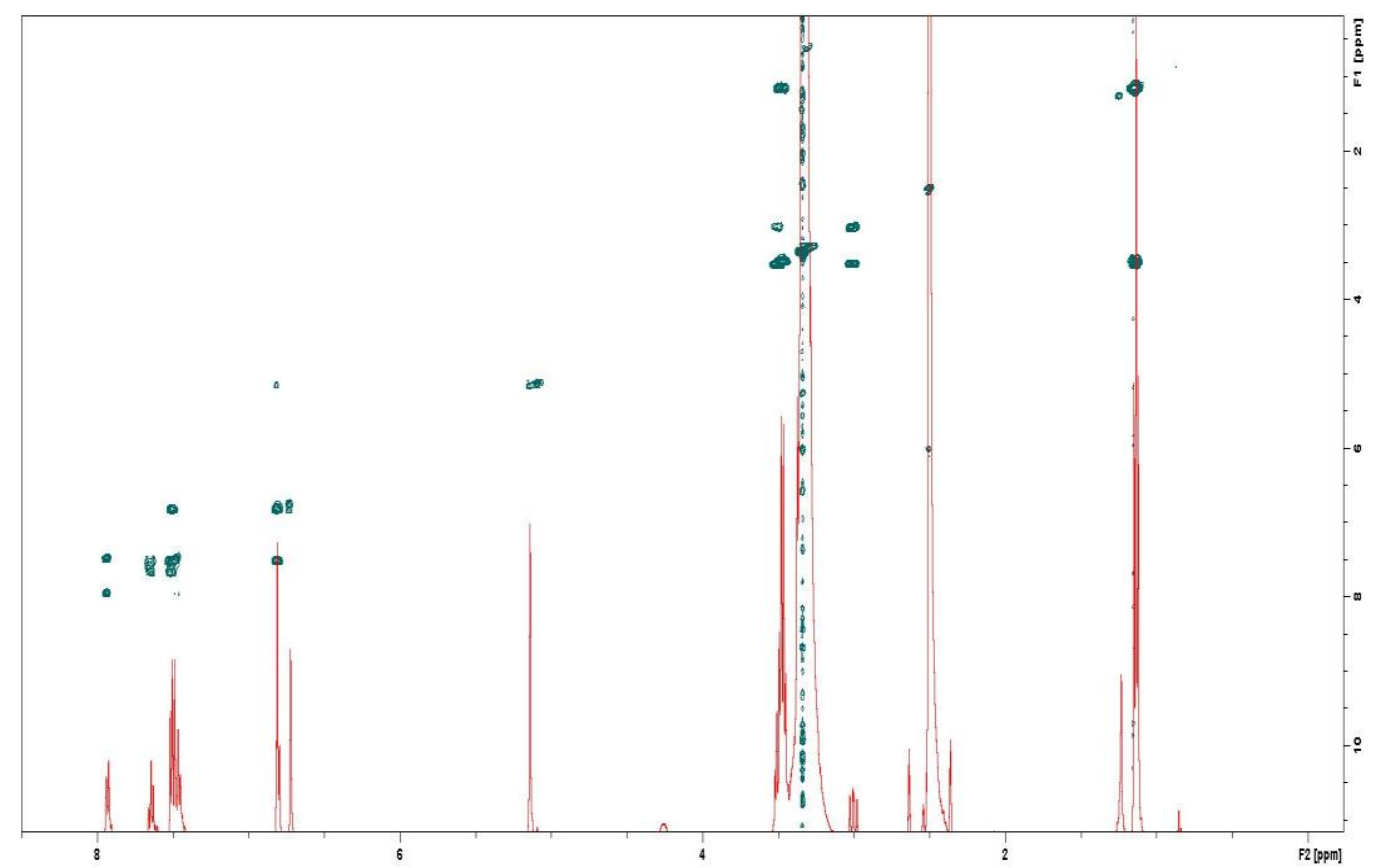

Figure S4. ${ }^{1} \mathrm{H}$ NMR-,${ }^{13} \mathrm{C}-{ }^{1} \mathrm{H}-\mathrm{HSQC}-,{ }^{1} \mathrm{H}-\mathrm{COSY}$ - and Mass (ESI) Spectra of thio-DEACM-nitrophenylalanine carbamate 9 .

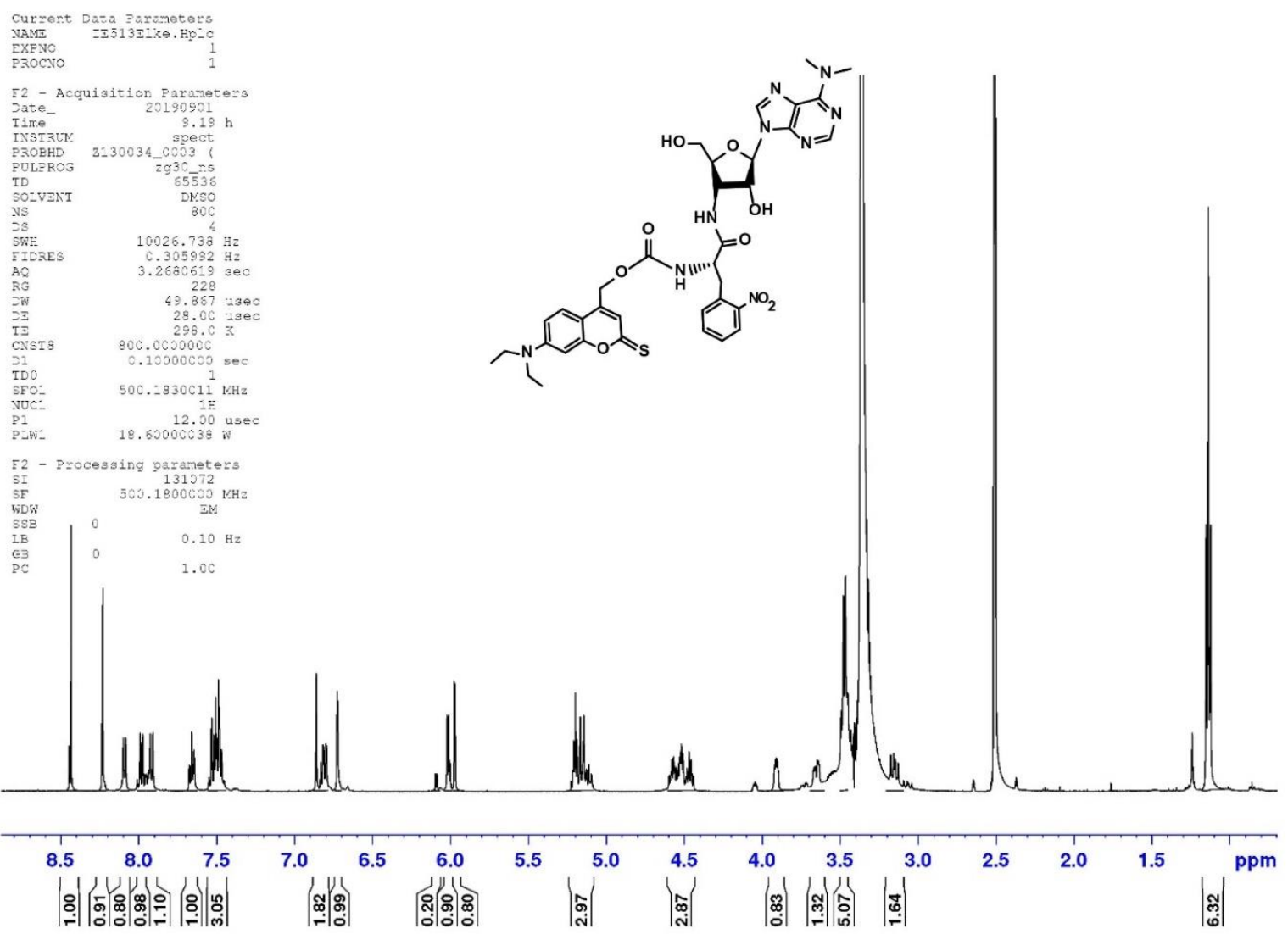



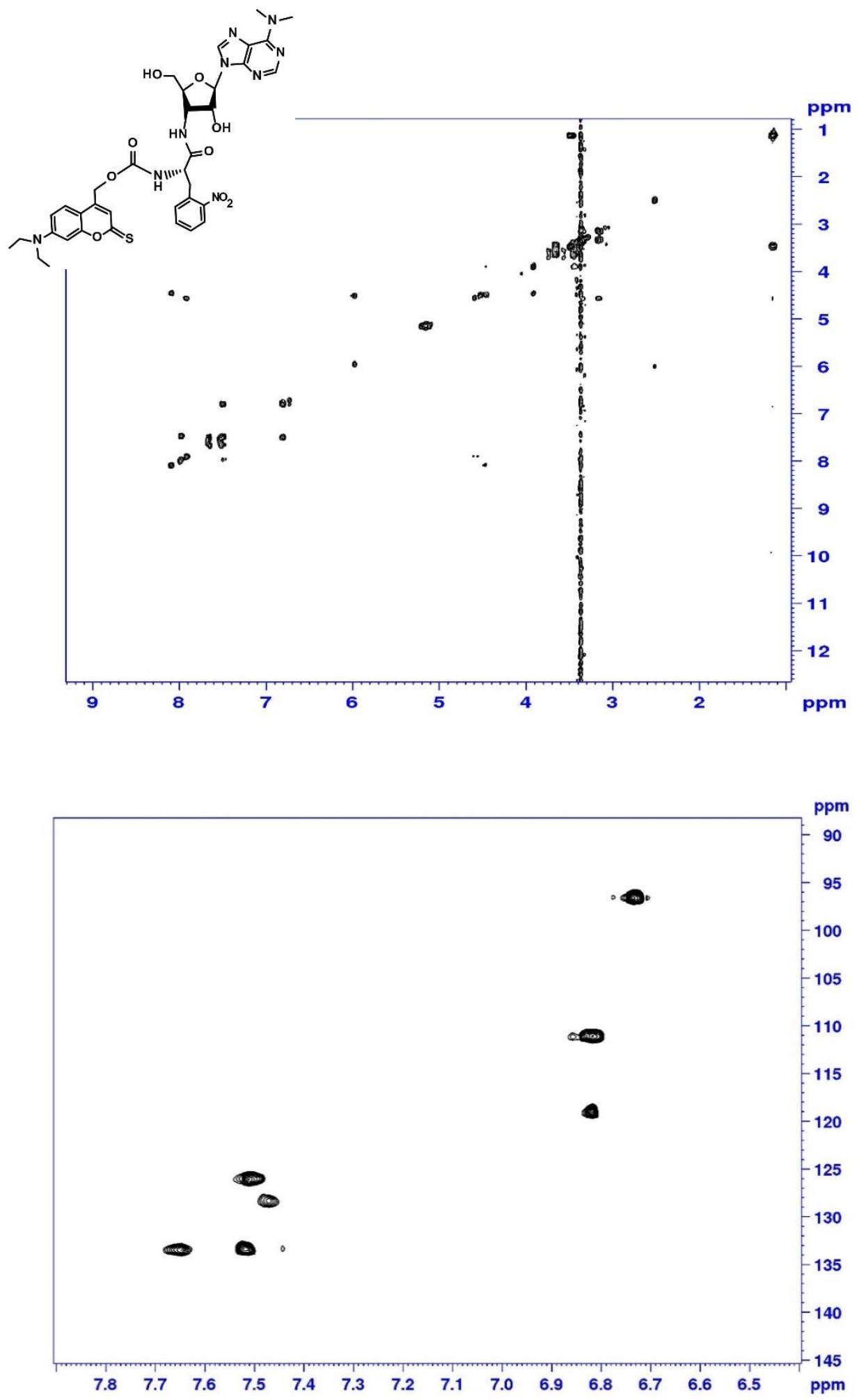


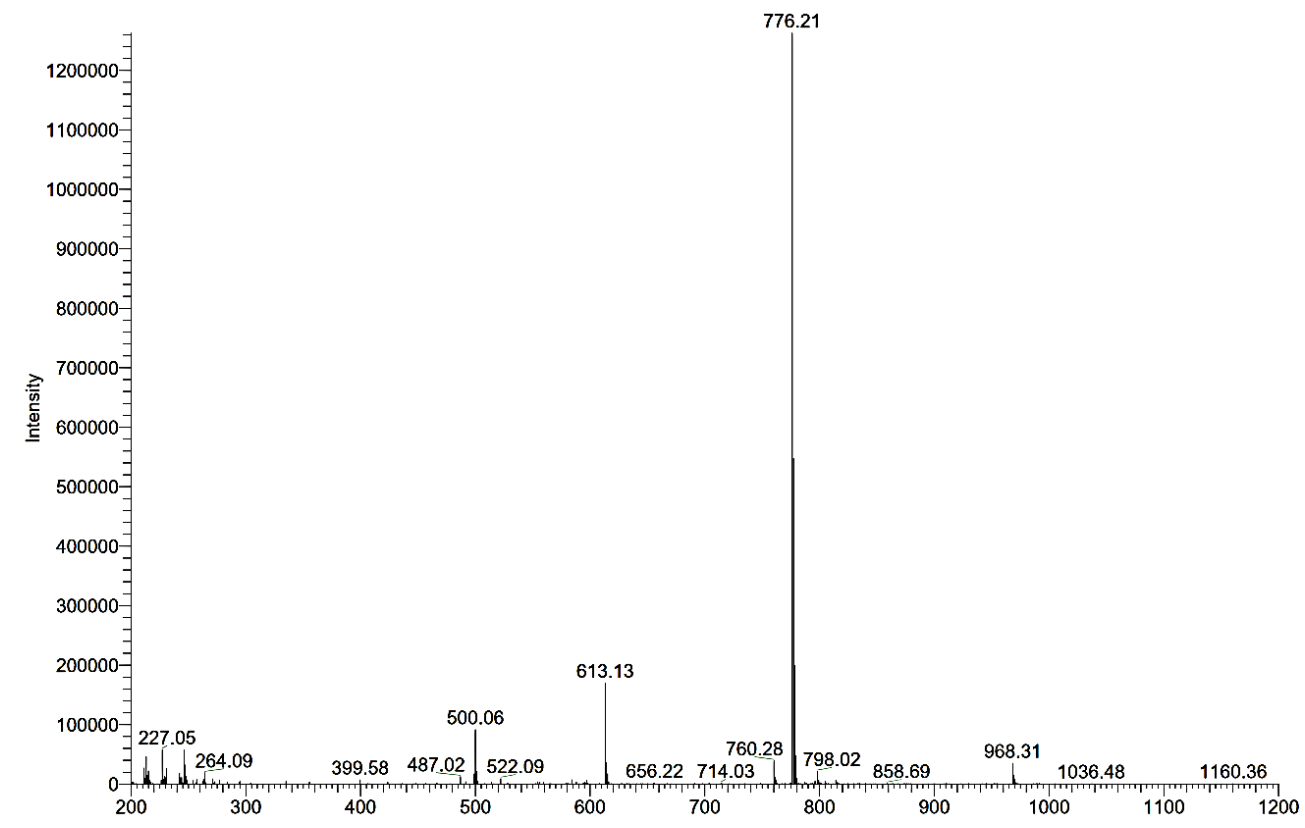

Figure S5. ${ }^{1} \mathrm{H}$ NMR-,${ }^{13} \mathrm{C}-{ }^{1} \mathrm{H}-\mathrm{HSQC}-,{ }^{1} \mathrm{H}-\mathrm{COSY}-$ and Mass (ESI) Spectra Caged thioDEACM-ortho-nitro-puromycin (I).
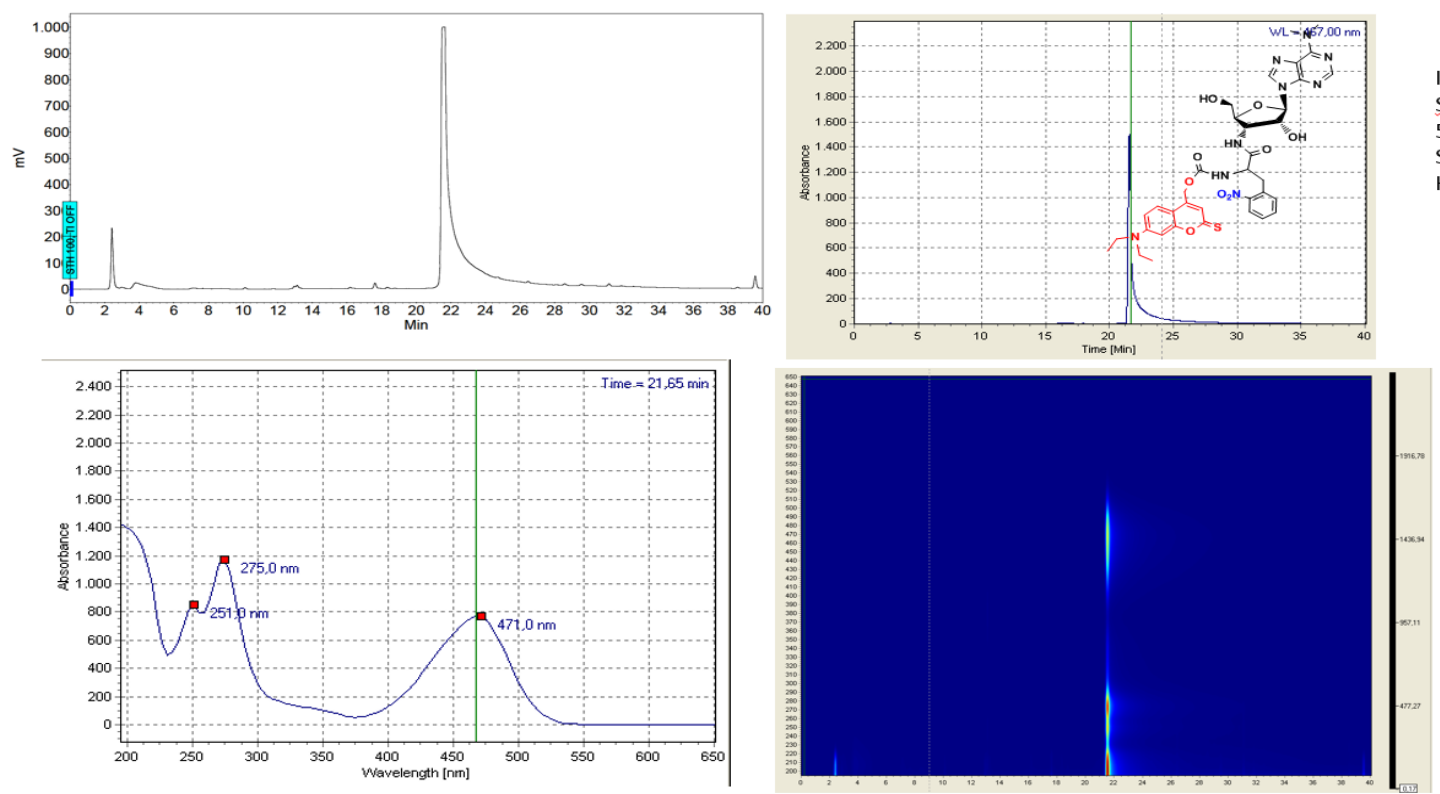

Figure S6. 1D and 2D-HPLC-chromatograms and absorption spectrum of thio-DEACMortho-nitro-puromycin (I). 

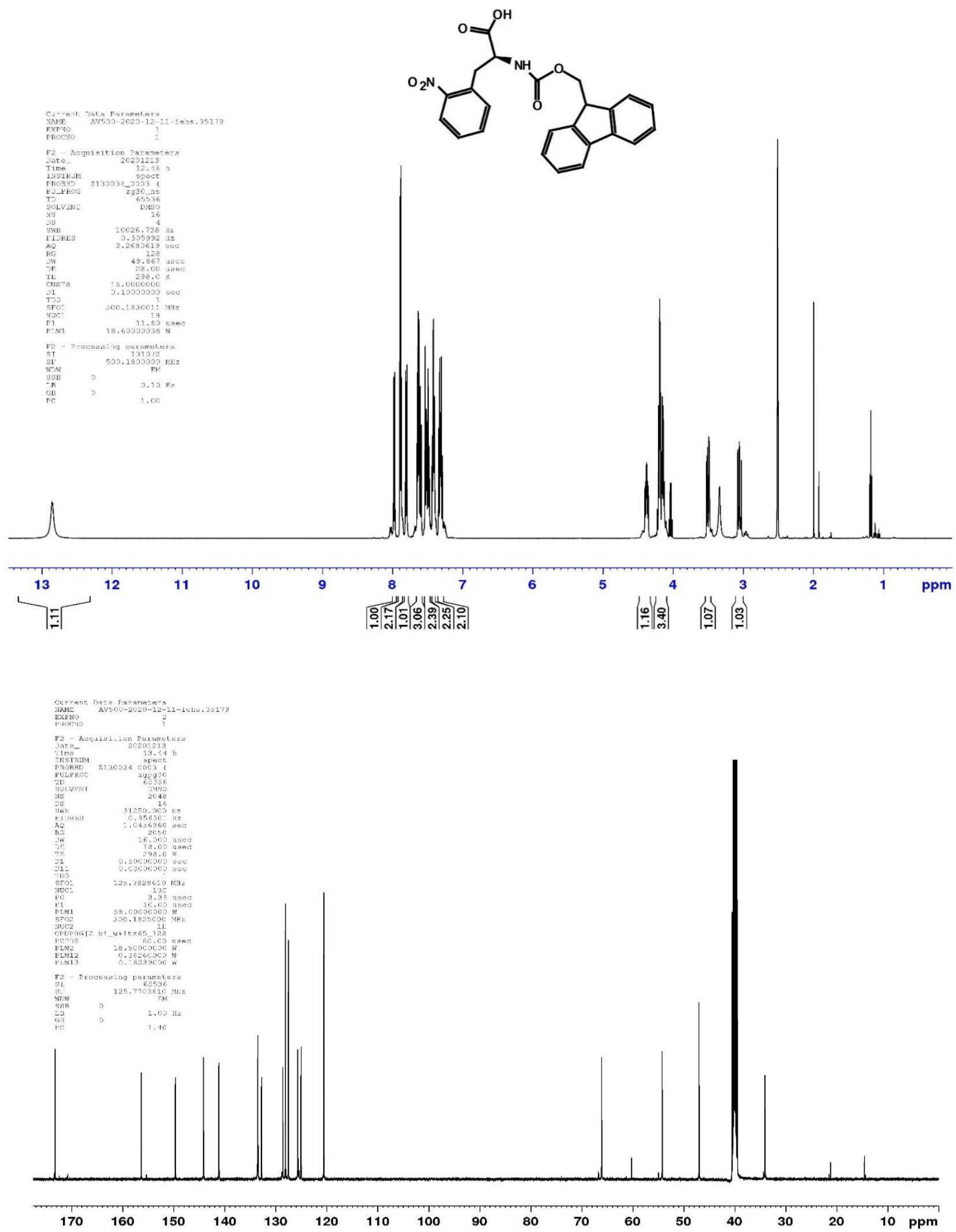

Figure S7. ${ }^{1} \mathrm{H}$ - and ${ }^{13} \mathrm{C}$-NMR spectra of FMOC-nitrophenylalanine. 

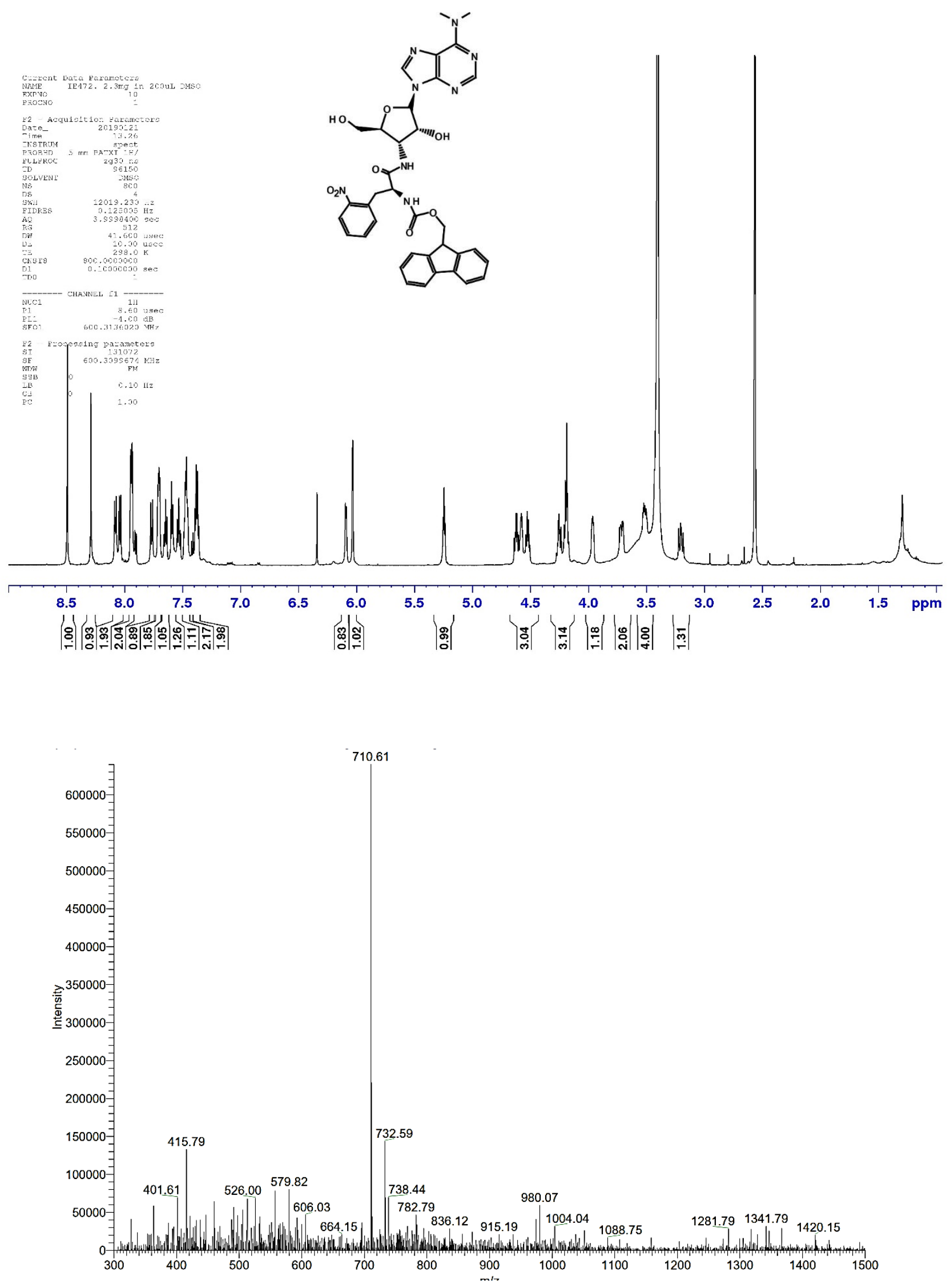


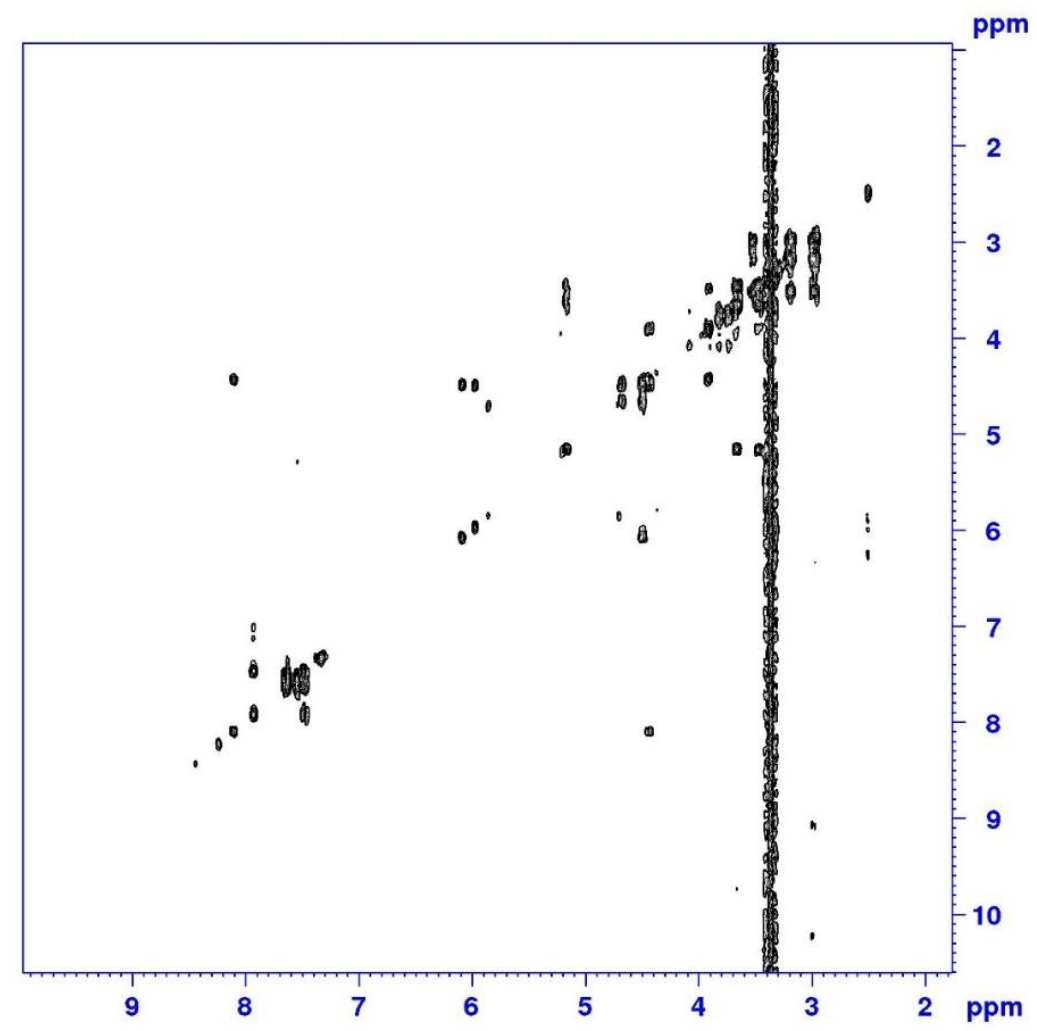

Figure S8. ${ }^{1} \mathrm{H}-,{ }^{1} \mathrm{H}-{ }^{1} \mathrm{H}-\mathrm{COSY}$ and Mass (ESI) spectra of FMOC-nitropuromycin (13). 

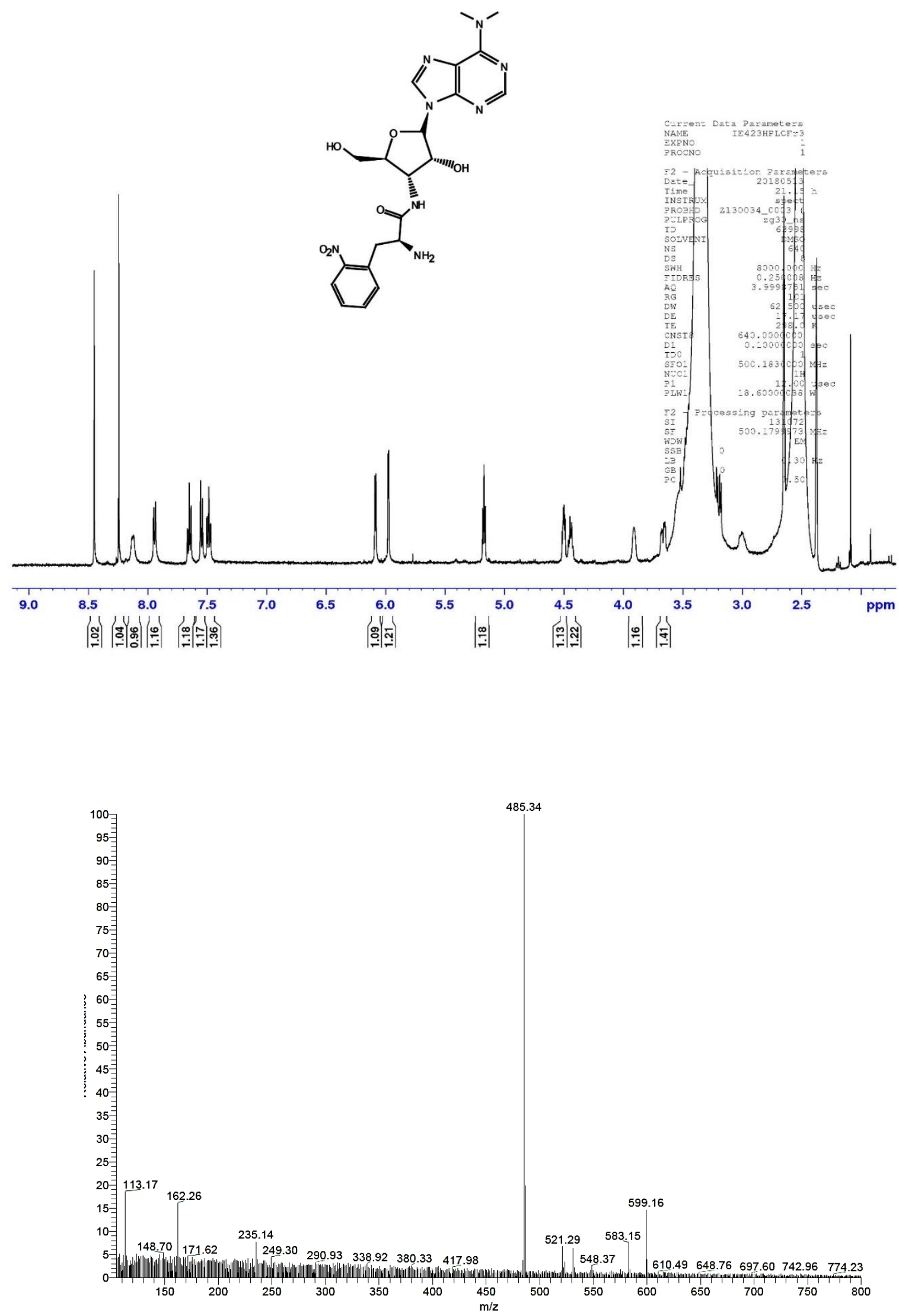


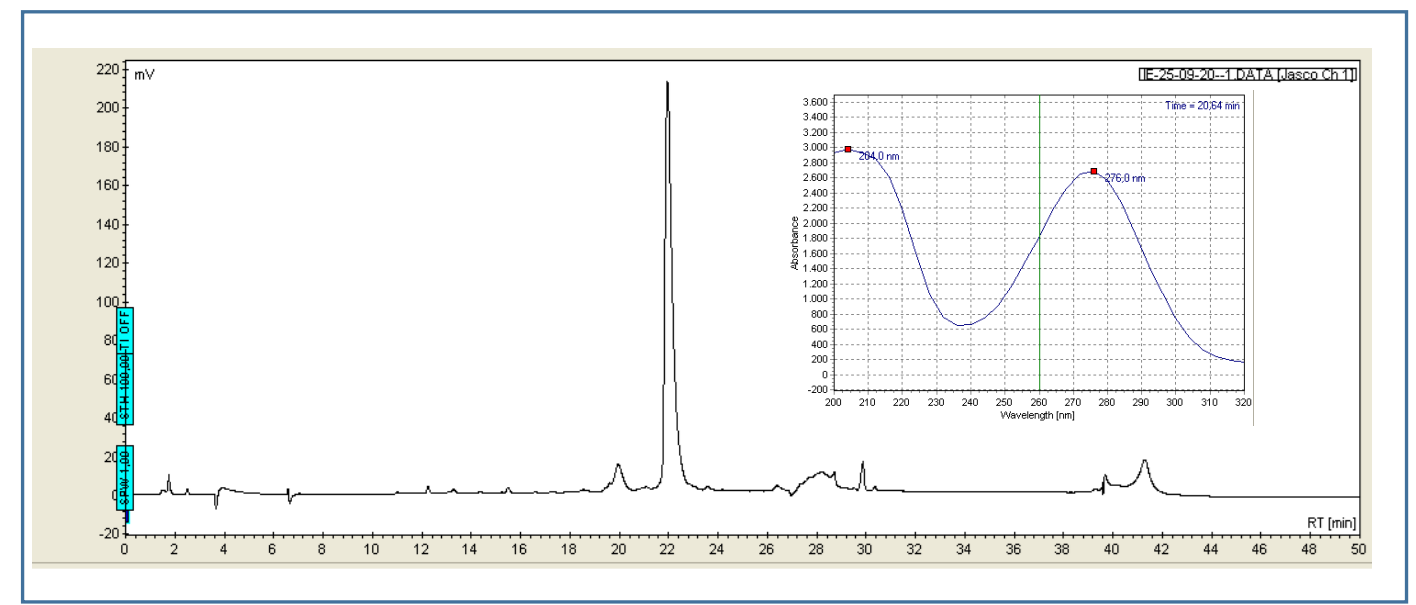

Figure S9. ${ }^{1} \mathrm{H}-\mathrm{NMR}$, Mass (ESI) and 1D chromatograms and absorption spectrum of orthonitro-puromycin (II).

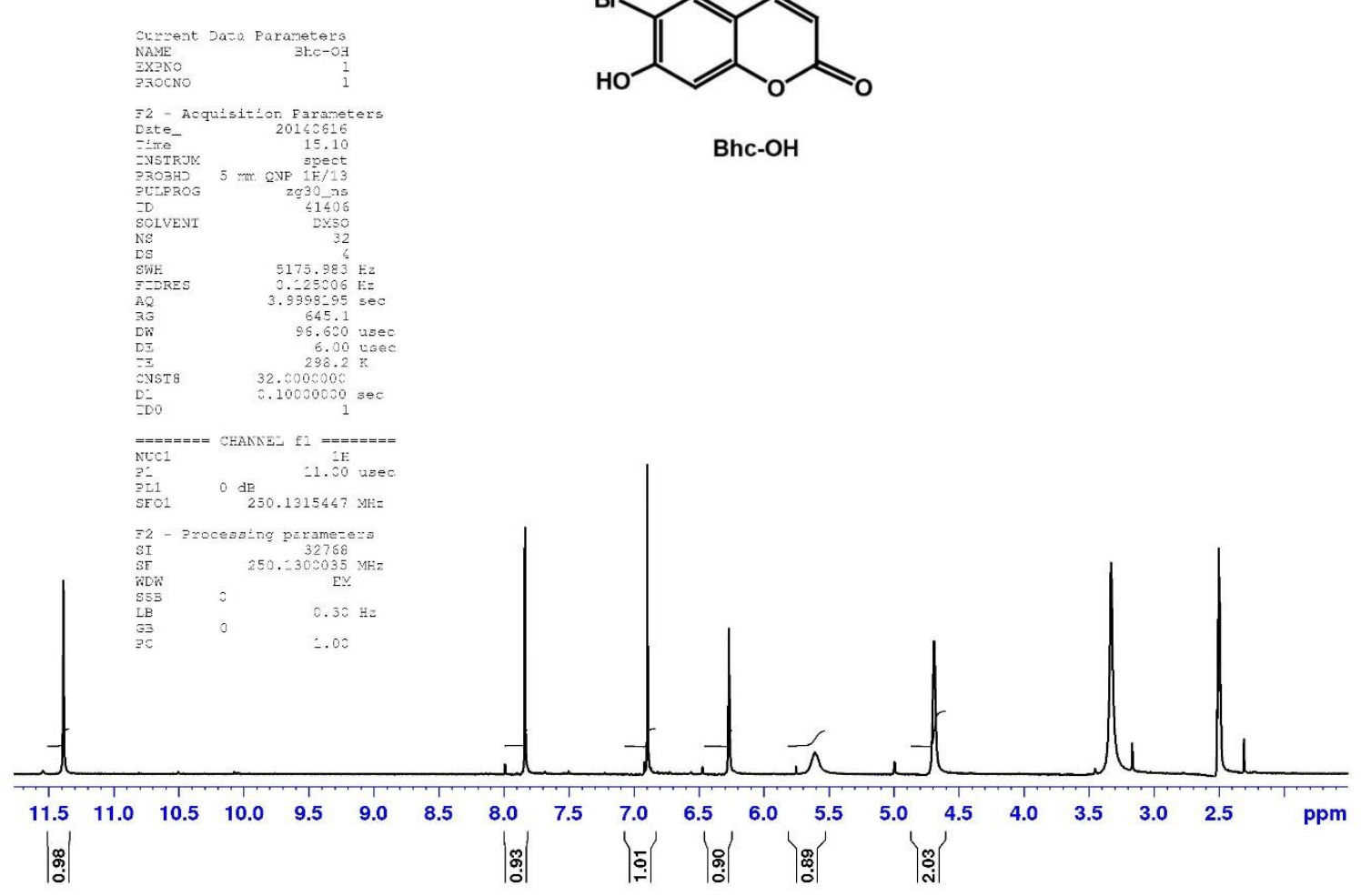

Figure S10. ${ }^{1} \mathrm{H}$ spectrum of Bhc-OH. 


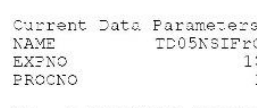

F2 - Acquisition Paramezers

Date-

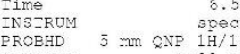

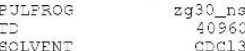

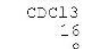

$\begin{array}{lr}\text { SWH } & 4990.020 \mathrm{~Hz} \\ \text { FIDRES } & 0.122 .827 \mathrm{~Hz} \\ \text { AQ } & 4.1041918 \mathrm{sec} \\ \text { RE } & 812.7 \\ \text { DW } & 100.200 \text { use } \\ \text { DE } & 10.09\end{array}$

$\begin{array}{lr}\text { FIDRES } & 4990.020 \mathrm{~Hz} \\ \text { AQ } & 0.12-327 \mathrm{Ez} \\ \text { RE } & 4.1041918 \mathrm{sec} \\ \text { DW } & 812.7 \\ \text { DE } & 100.200 \text { use } \\ \text { DE } & 10.05 \text { use }\end{array}$

$\begin{array}{lr}\mathrm{DE} & 10.00 \text { use } \\ \mathrm{ZE} & 300.2 \mathrm{~K}\end{array}$

$\begin{array}{ll}\text { CNSZ8 } & -6.0000000 \\ \text { D1 } & 0.10000000 \mathrm{se}\end{array}$

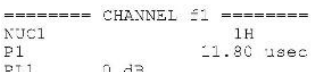

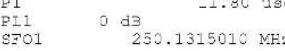

F2 - Procesaing parameters

$250.1300285 \mathrm{MHz}$

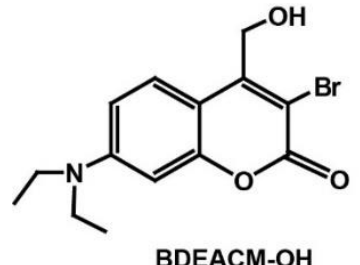

BDEACM-OH

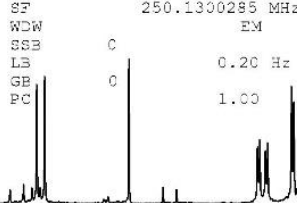

(1)

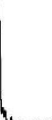

$\begin{array}{ll}7.5 & 7.0\end{array}$

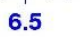

$6.0 \quad 5.5$

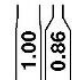

$\left|\begin{array}{c}0 \\ 0 \\ 0\end{array}\right|$

$|\stackrel{\infty}{\circ}|$

$4.5 \quad 4.0$

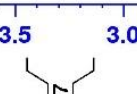

กิ

ppm

월

Figure S11. ${ }^{1} \mathrm{H}$ spectrum of BDEACM-OH. 


\section{Photolysis control experiments}

Nitrophenylalanine and phenylalanine
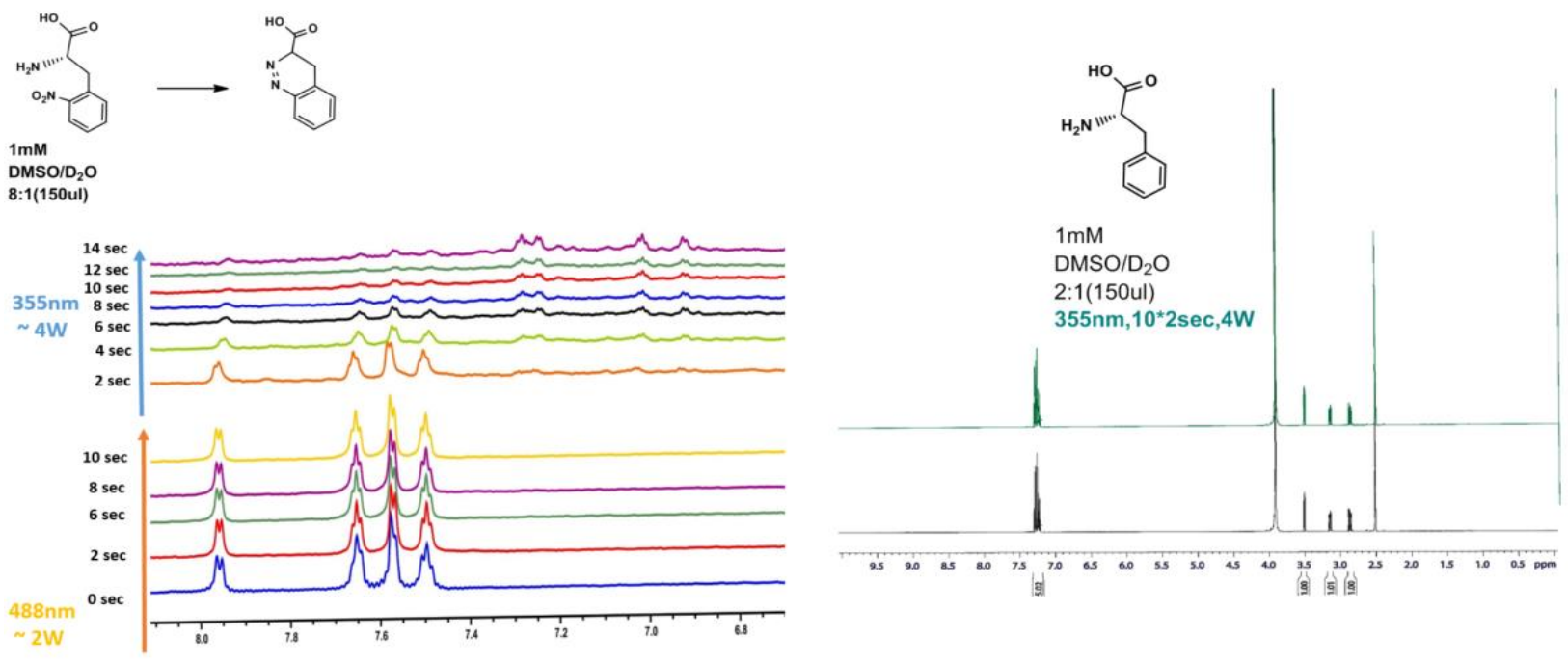

Figure S12. Monitoring of photolysis of ortho-nitrophenylalanine and no-photolysis of phenylalanine by using Laser-coupled NMR experiment at 488- and $355 \mathrm{~nm}$.

Thio-DEACM-ortho-nitro-puromycin (I)
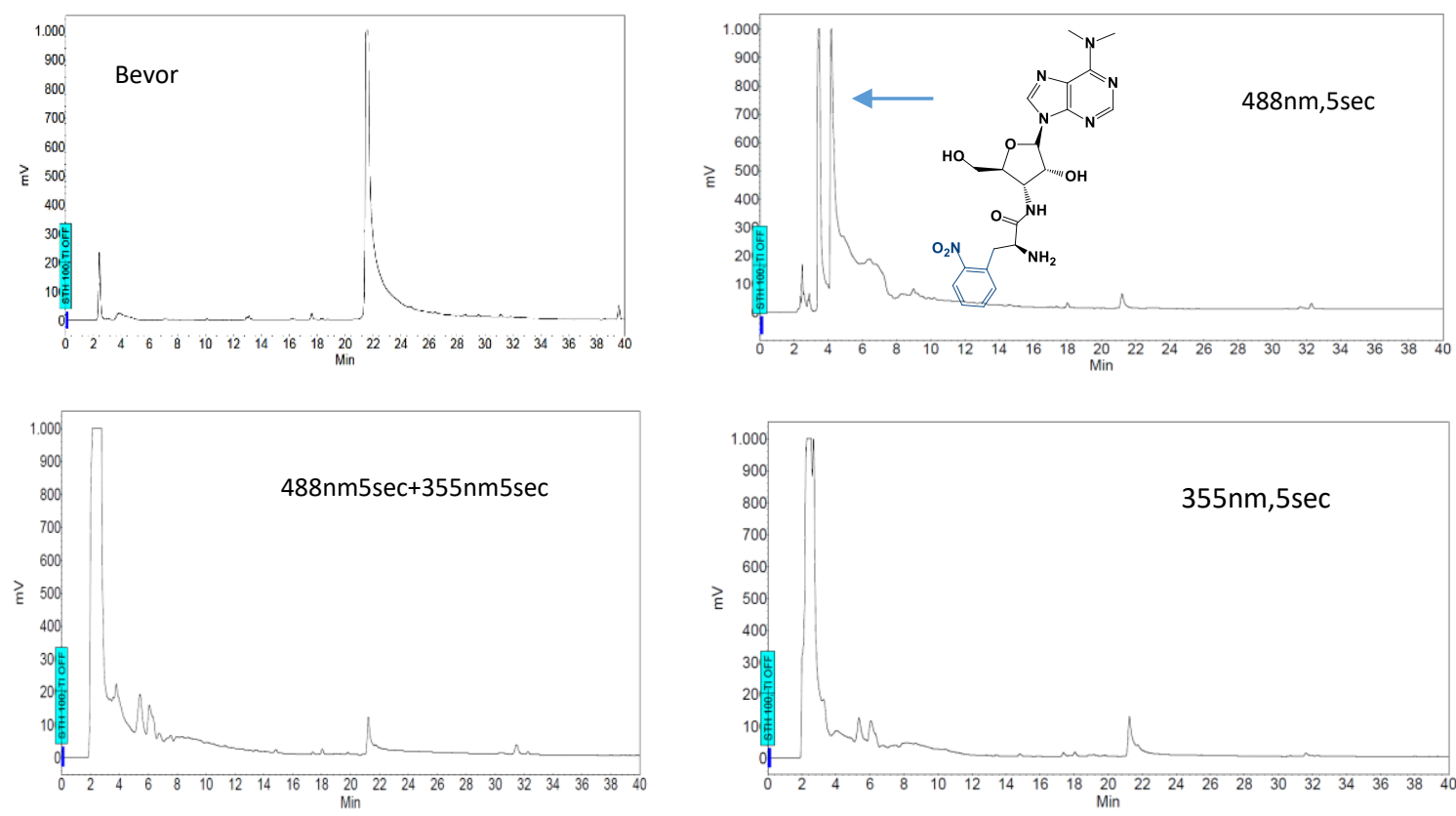

Figure S13. Monitoring of photolysis of thio-DEACM-ortho-nitro-puromycin (I) by using rp$\mathrm{HPLC}\left(\mathrm{H}_{2} \mathrm{O} / \mathrm{ACN}, 0-100 \% 50 \mathrm{~min}\right)$ upon Laser irradiation at 488- and $355 \mathrm{~nm}$. 
Photolysis of thio-DEACM-OH
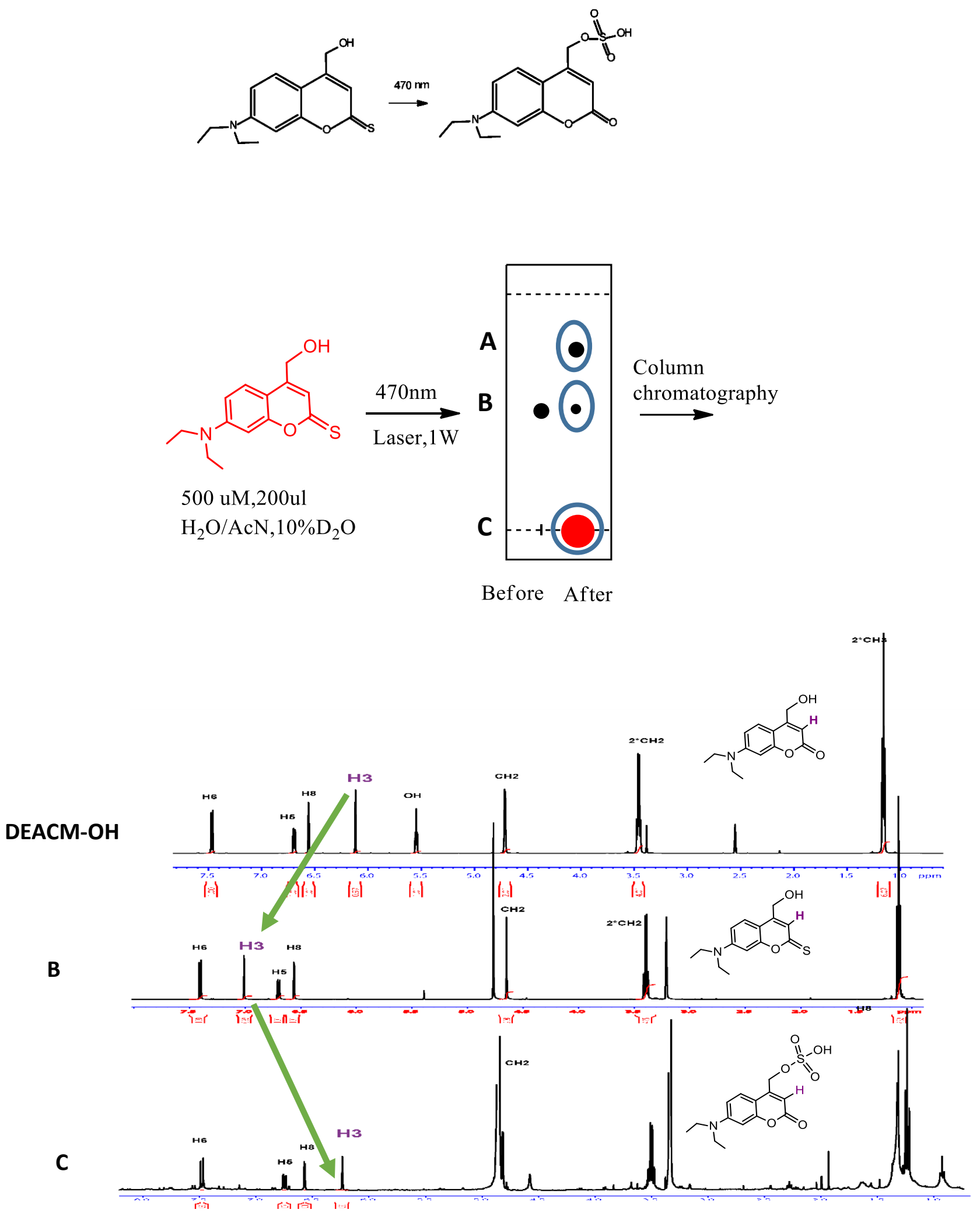


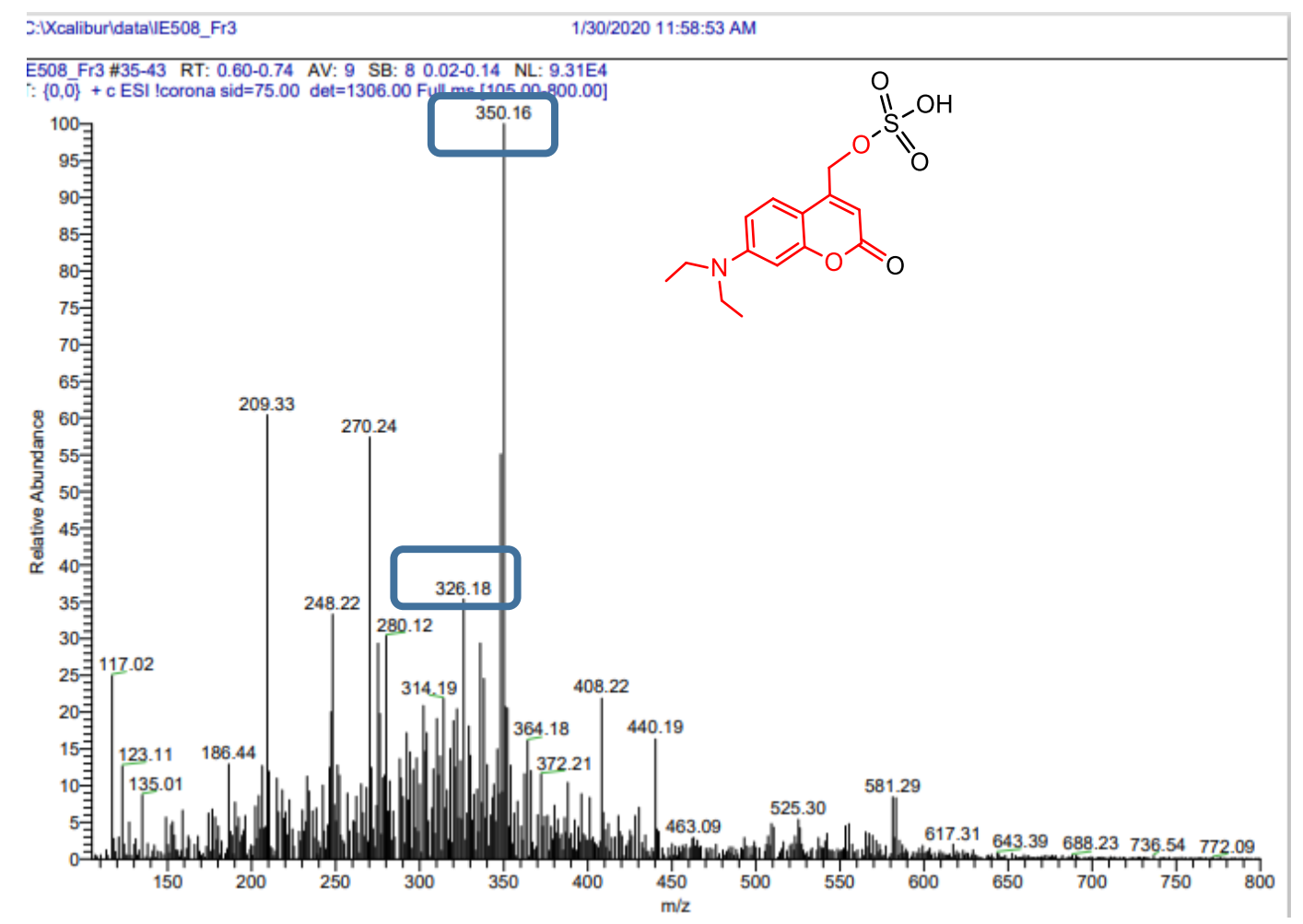

Figure S14. Identification of sulferic acide coumarylmethyl ester (Fraction C) as the main photo-product upon photolysis of thio-DEACM by rp-HPLC purification and ${ }^{1} \mathrm{H}-\mathrm{NMR}$ - and mass spectroscopy. The chemical shift of $\mathrm{H}-3$ indicated the reoxidation of thio-DEACM (Fraction B), (DEACM-OH as reference). Fraction A showed a mixture of several compounds with negligible concentrations. 


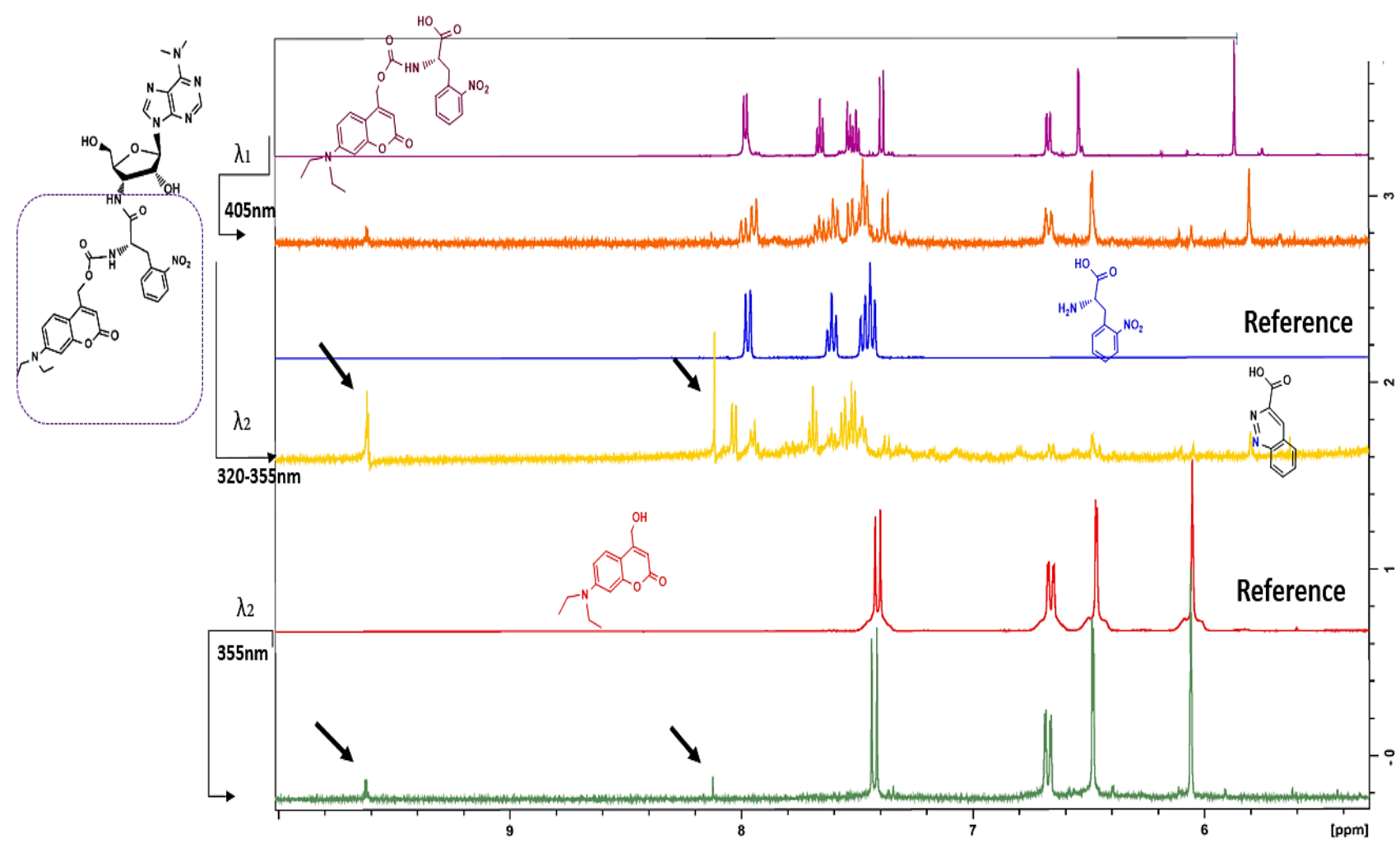

Figure S15. Demonstration of the unsuitability of DEACM-OH for a wavelength selective photolysis of DEACM-OH and ortho-nitrophenylalanine: The irradiation of DEACM-nitrophenylalanine carbamate (9, purple spectrum) at $405 \mathrm{~nm}$ to remove DEACM-OH as well at 355 $\mathrm{nm}$ to induce the photocyclization of nitro- phenylalanine (blue spectrum) lead to the formation of a by-product ( 8.1 and $9.4 \mathrm{ppm}$ ). Noteworthy, the illumination of DEACM-OH (red spectrum) in separate experiment at $355 \mathrm{~nm}$ showed that formation of the same compound (8.1 and 9.4 ppm) occurring with a reaction rate that was much less compared with the photolysis of DEACM-nitro- phenylalanine carbamate (9, green spectrum). 


\section{Experimental details of Spectroscopic part}

The sample was first constantly illuminated with LEDs (Thorlabs $\mathrm{GmbH}$ ) at $470 \mathrm{~nm}$ and subsequently at $365 \mathrm{~nm}$. For the UV/vis measurements the spectra were recorded with a Specord S600 spectrophotometer (Analytik Jena) every $30 \mathrm{~s}$ for the first hour, afterwards every $60 \mathrm{~s}$. The IR spectra were recorded with a FTIR spectrometer Vertex 80 (Bruker, Ettlingen) every $60 \mathrm{~s}$ under constant nitrogen flow. All quantum chemical calculations were examined with Gaussian09. For the UV/vis transitions the ground state geometries were optimized on the theory level DFT/B3Lyp with the basis set cc-pVDZ. The vibrational modes were calculated, while theory level DFT/BP86 were used in combination with SVP as basis set.

Determination of quantum yields

The quantum yields were determined by the time dependent absorption change of a species $(\mathrm{m})$ with known extinction coefficient $(\varepsilon)$ and from the increase/decrease of absorbance. The quantum yield can be calculated using the output power $\left(\mathrm{P}_{0}\right)$ of the applied light source $(\lambda)$ :

$$
\Phi=\frac{m \cdot N_{A} \cdot V}{\varepsilon \cdot d} \frac{h \cdot c}{P_{0} \cdot \lambda \cdot\left(1-10^{A_{0}}\right)}
$$

Absorptions spectra of the doubly caged compound thio-DEACM-ortho-nitropuromycin and its components

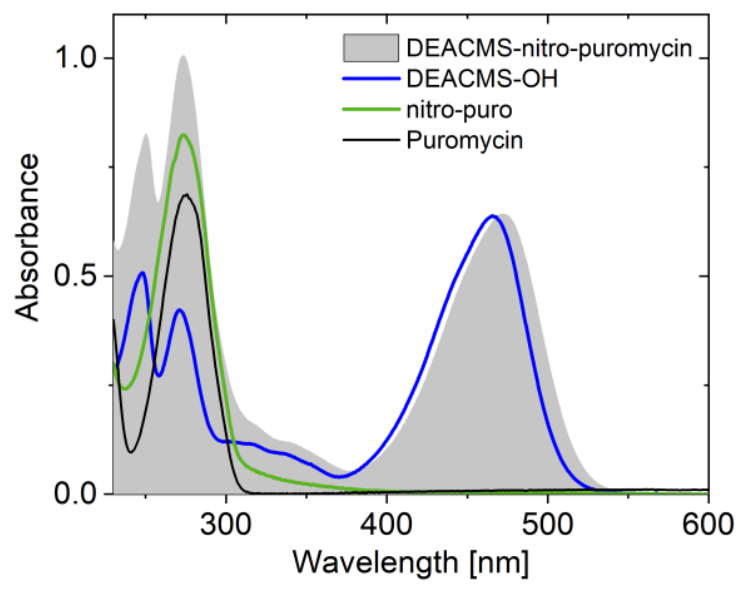

Figure S16. Absorption spectra of thio-DEACM-ortho-nitro-puromycin (I) (grey) and its components thio-DEACM-OH (blue), ortho-nitro-puromycin (II) (green) and puromycin (black). 
Investigation of thio-DEACM cage and its photolysis products

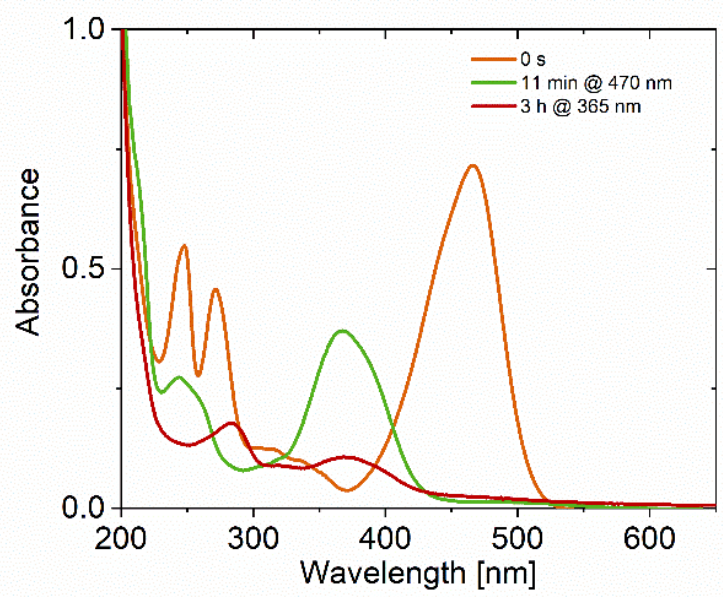

Figure S17. Absorption spectra of thio-DEACM-OH (7) before (orange) and after illumination with $470 \mathrm{~nm}$ for $11 \mathrm{~min}$ (green) and with $365 \mathrm{~nm}$ for $3 \mathrm{~h}$ (red) in a 1:1 ACN/ $\mathrm{H}_{2} \mathrm{O}$ mixture.

a

b
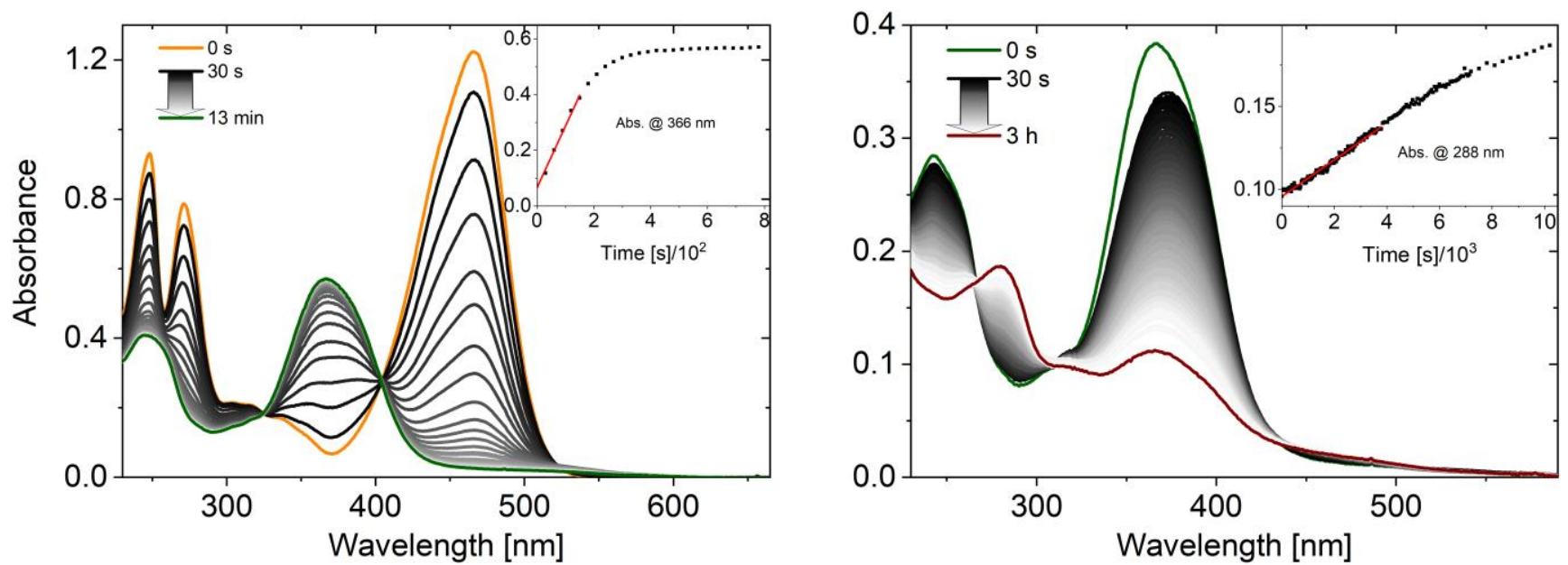

Figure S18. Absorption spectra of thio-DEACM-OH (7) during illumination with $470 \mathrm{~nm}$ (a) and with $365 \mathrm{~nm}$ (b) and the fits of the newly formed absorption bands. 

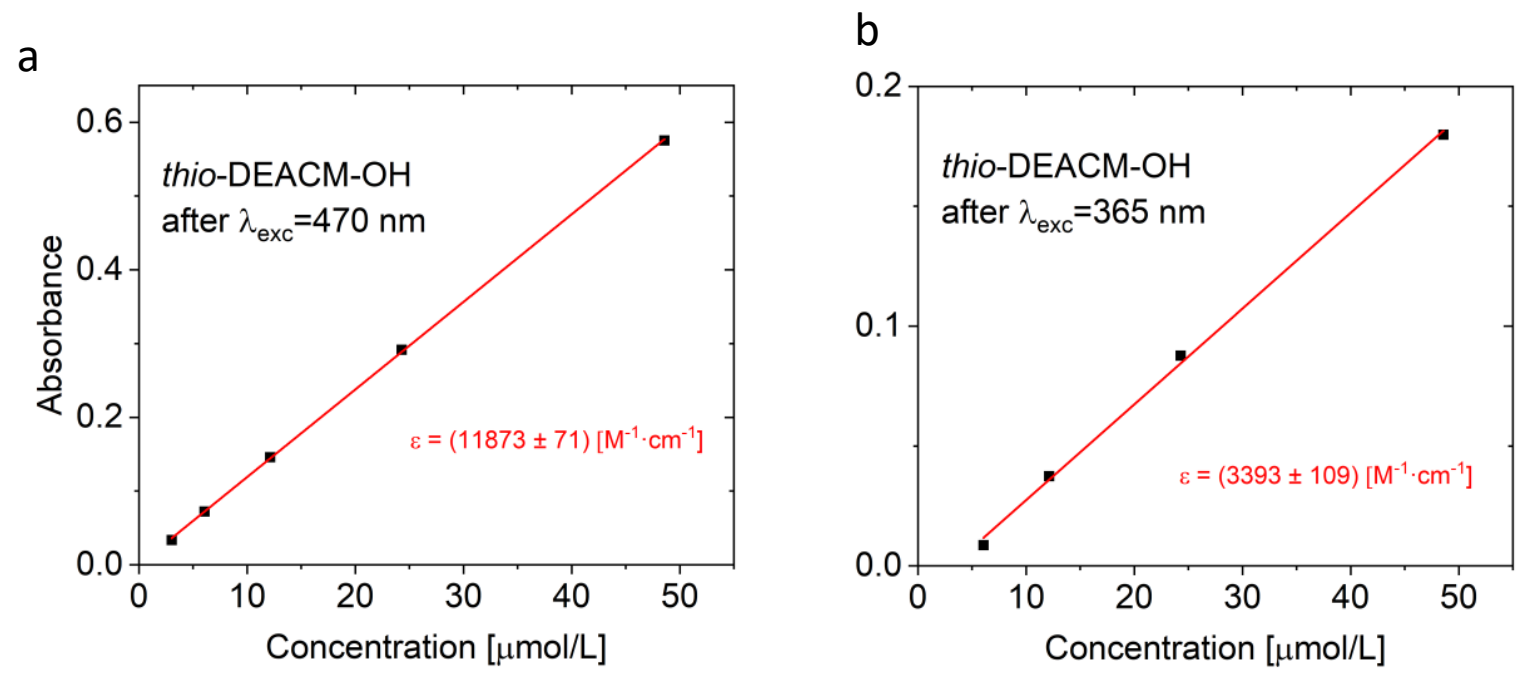

Figure S19. Determination of molar extinction coefficients of photolysis products of thioDEACM-OH (7) after illumination at $470 \mathrm{~nm}$ (a) and $365 \mathrm{~nm}$ (b).

Spectral changes of nitro-puromycin upon illumination

a

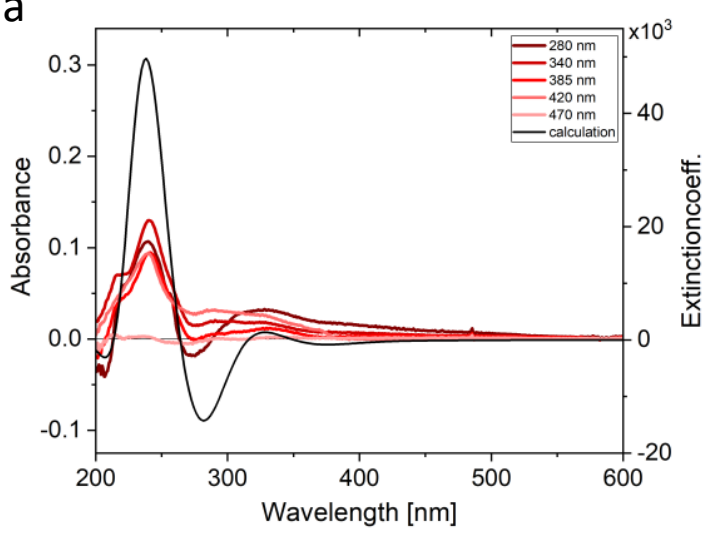

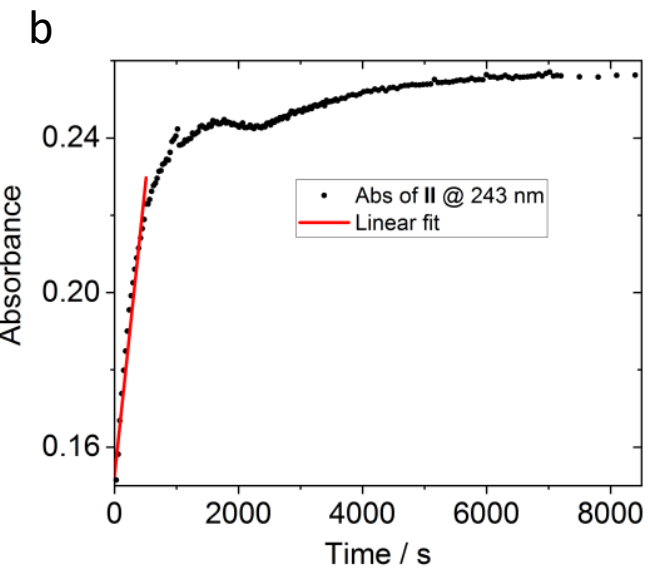

Figure S20. (a) Difference spectra of ortho-nitro-puromycin (II) after illumination with different wavelengths from $280 \mathrm{~nm}$ to $470 \mathrm{~nm}$ (red) and the DFT-calculated difference spectrum of nitro-puromycin and the photo-cyclized cinnoline product (black). (b) Transient of II at $243 \mathrm{~nm}$ during illumination with $340 \mathrm{~nm}$ used to calculate the reaction quantum yield ( $\varphi$ No2-puro $=2.5 \%$ ). 


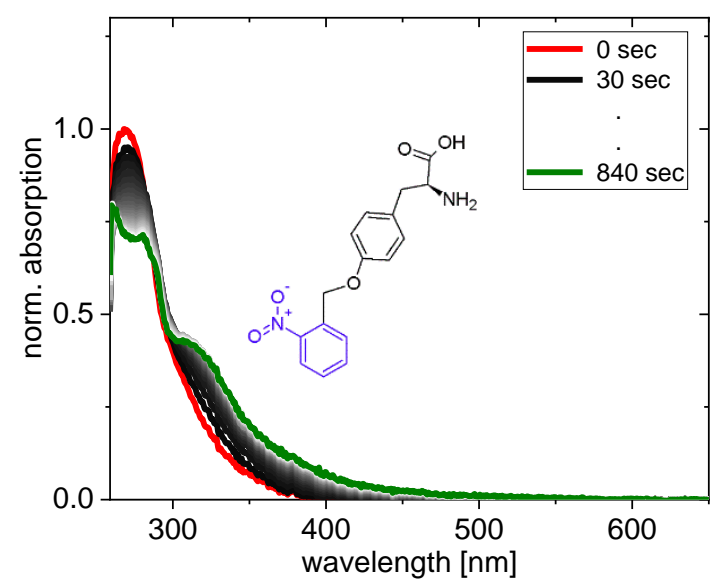

Figure S21. Absorption spectra before (red), during (black to grey) and after (green) illumination of $o$ NB-tyrosine in DMSO at $340 \mathrm{~nm}$.

\section{Direct deactivation of thio-DEACM-ortho-nitro-puromycin}
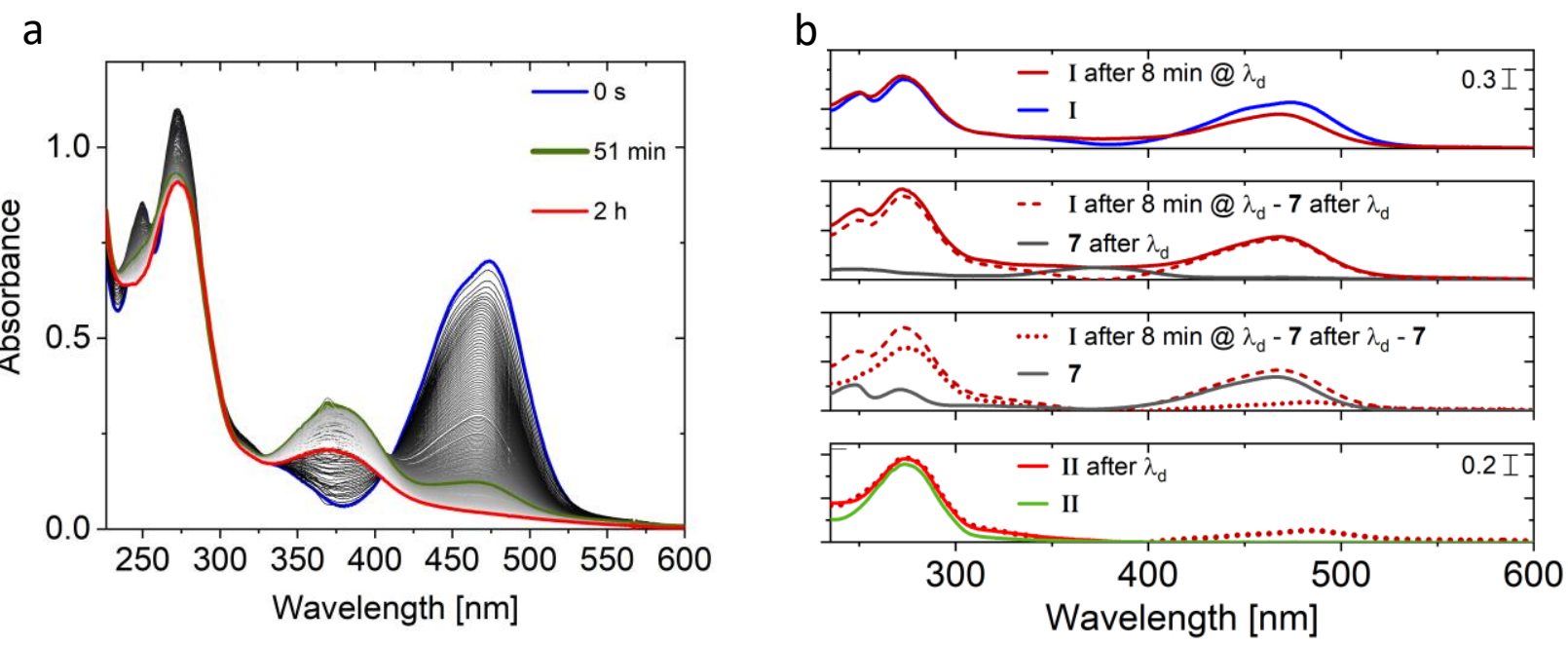

Figure S22. Absorption spectra of thio-DEACM-ortho-nitro-puromycin (I) during illumination with $365 \mathrm{~nm}$ (a) and spectrum of the same experiment after 8 minutes of illumination (dark red solid line) from which all photoproducts of both uncaging step were subtracted step by step. The final spectrum shows that not only the activation is performed which is clear due to the loss of the $470 \mathrm{~nm}$ band but also the deactivation step of nitro-puromycin (II) (see overlap with the spectrum of separately illuminated nitro-puromycin (II) (red solid line)) (b). 


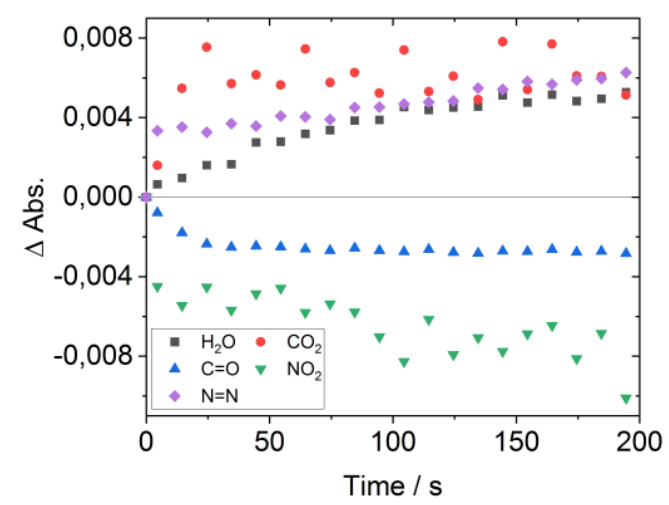

Figure S23. IR-transients of thio-DEACM-ortho-nitro-puromycin (I) during illumination at $365 \mathrm{~nm}$ directly. Changes of characteristic vibrational modes of both activation and deactivation are observable during $365 \mathrm{~nm}$ illumination: $\mathrm{H}_{2} \mathrm{O}: 3608 \mathrm{~cm}^{-1}$ (black squares), $\mathrm{CO}_{2}: 2337 \mathrm{~cm}^{-1}$ (red dots), $\mathrm{C}=\mathrm{O} 1729 \mathrm{~cm}^{-1}$ (green triangle), cinnoline moiety: $1398 \mathrm{~cm}^{-1}$ (violet rhombs), $\mathrm{CO}$ $1141 \mathrm{~cm}^{-1}$ (blue triangles).

\section{Spectral changes of DEACM-ortho-nitro-puromycin}

a

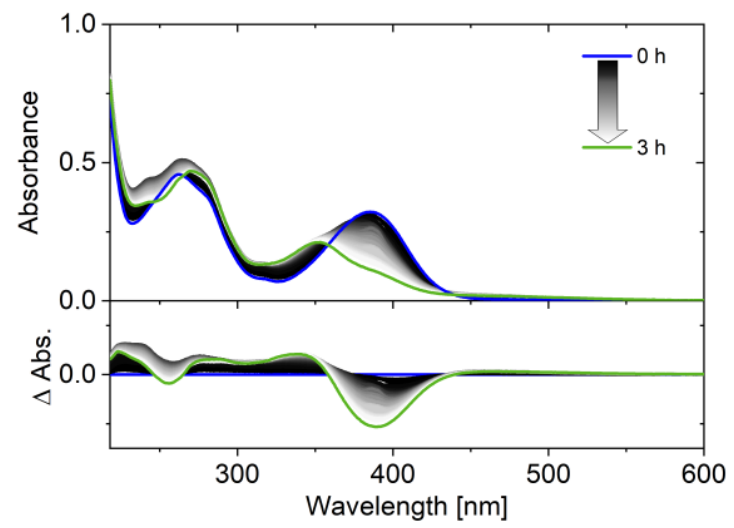

b

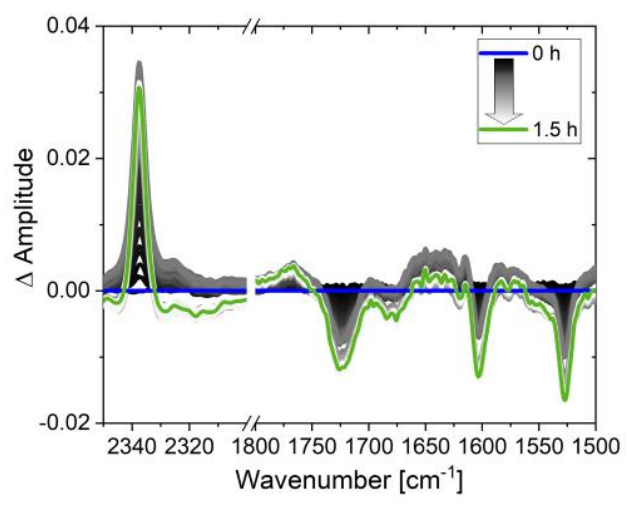

Figure S24. UV/vis-absorption spectra of DEACM-ortho-nitro-puromycin during illumination with $380 \mathrm{~nm}$ (a). IR-difference spectra of DEACM-ortho-nitro-puromycin during illumination with $380 \mathrm{~nm}(\mathrm{~b})$. 


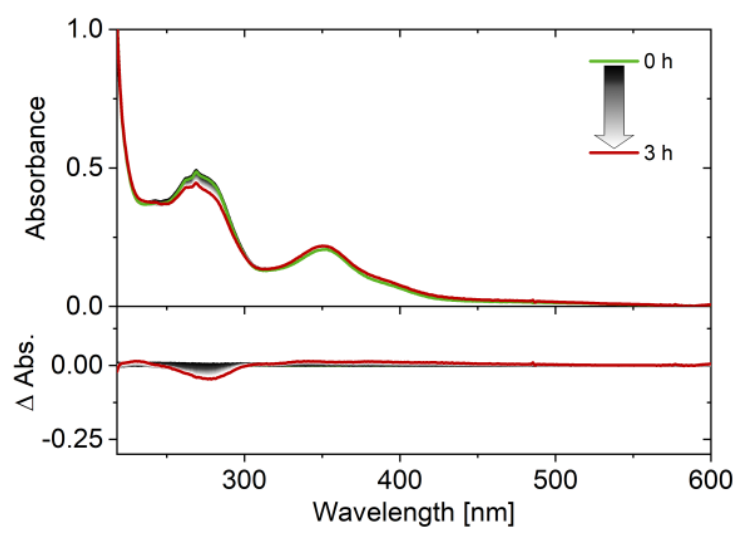

Figure S25. UV/vis-absorption spectra of DEACM-ortho-nitro-puromycin during illumination with $280 \mathrm{~nm}$ after the previous illumination with $380 \mathrm{~nm}$. Almost no absorption change is observable after 3 hours.

Spectral changes of thio-DEACM-ortho-nitro-puromycin in methanol / water
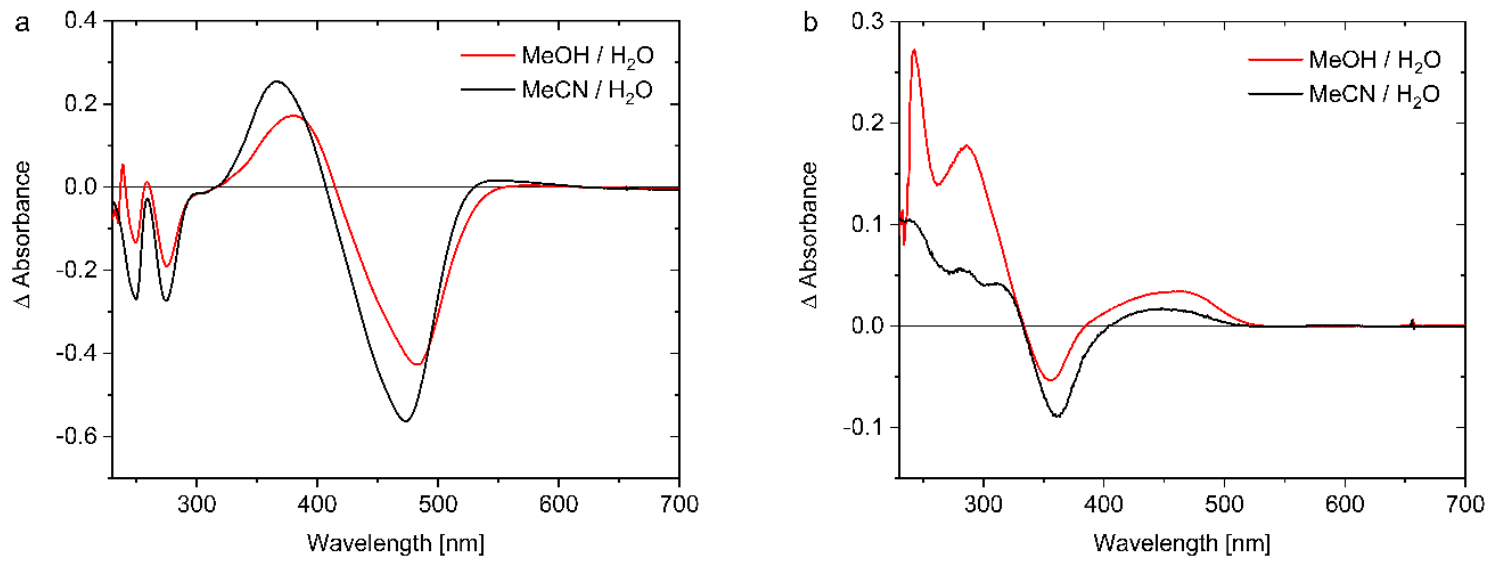

Figure S26. Difference spectra of thio-DEACM-o-nitro-puromycin (I) in 1:1 acetonitrile / water mixture (black) and 1:1 methanol / water mixture (with $0.5 \%$ DMSO for solubility) (red) after 5 minutes of illumination at $470 \mathrm{~nm}$ (a) and at $365 \mathrm{~nm}$ (b) of the completely activated sample. As can be seen the doubly caged puromycin shows hardly any differences in activation and deactivation in a protic solvent like methanol instead of acetonitril, except for slightly shifted absorption bands. 
In vitro transcription-translation essay

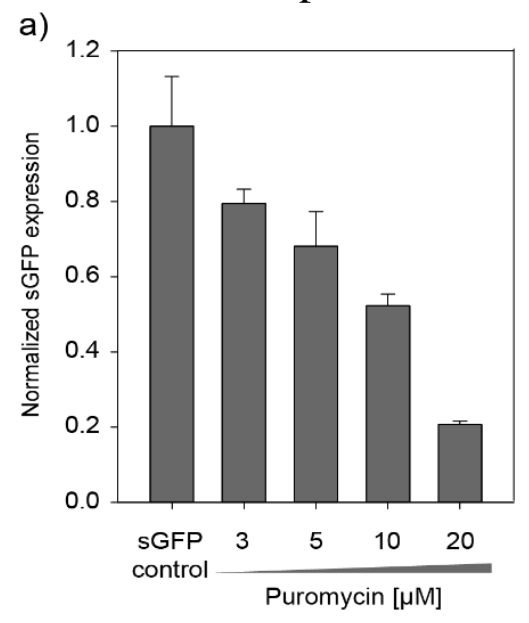

b)

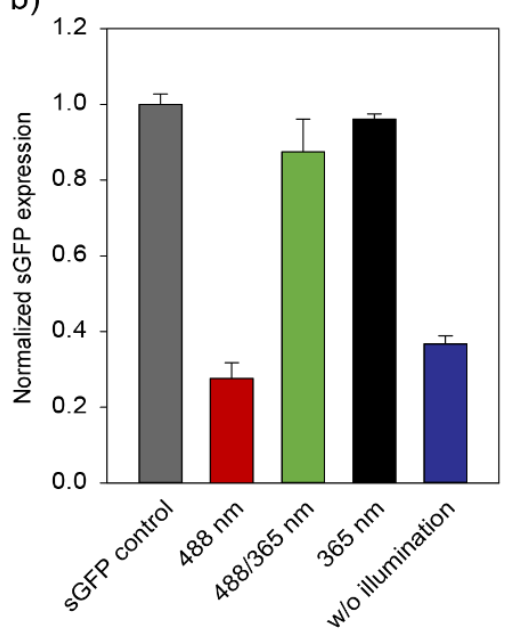

c)

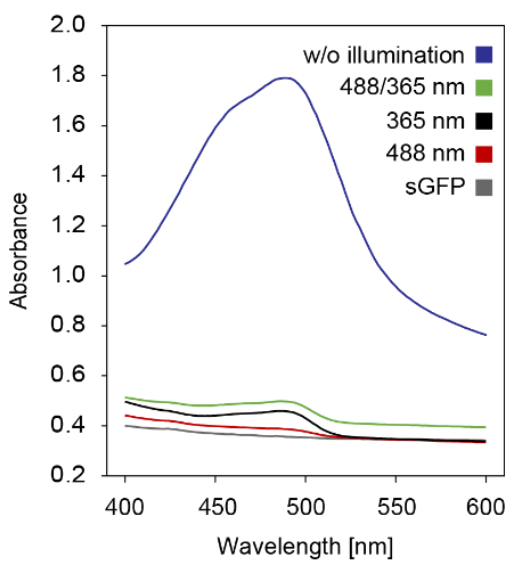

Figure S27. a) Inhibition of sGFP expression with increasing puromycin concentration. $20 \mu \mathrm{M}$ puromycin results in a 5-fold inhibition of protein expression. b) Thio-DEACM-nitropuromycin I was illuminated, respectively with LED at $365 \mathrm{~nm}, 365 / 488 \mathrm{~nm}, 488 \mathrm{~nm}$ and the effects of the illumination wavelength were tested by measuring the fluorescence of sGFP. c) Absorption spectra measured after finished sGFP expression.

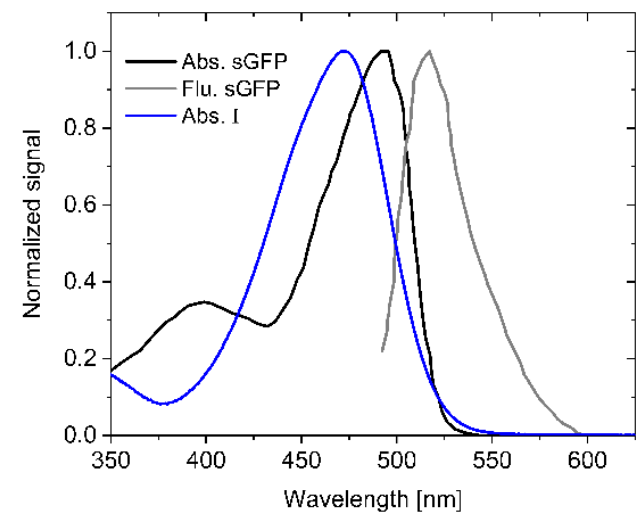

Figure S28: Absorption and fluorescence spectra of sGFP. Thio-DEACM-nitro-puromycin was illuminated, respectively with LED at $365 \mathrm{~nm}, 365 / 488 \mathrm{~nm}, 488 \mathrm{~nm}$ and the effects of illumination wavelengths were tested on sGFP expression by using a coupled in vitro transcription-translation assay. Surprisingly, the non-illuminated TPOM results in a lower sGFP expression, when measuring the fluorescence intensity. In addition, the attempt to excite sGFP with light in the range between $350 \mathrm{~nm}$ and $450 \mathrm{~nm}$ was unsuccessful too, the GFP signal was too weak. Thio-DEACM was absorbing the excitation/emission light due to the spectral overlap of thio-DEACM-nitro-Puromycin and sGFP. Further, we verified our results with native PAGE analysis, which showed no inhibition of sGFP expression with non-illuminated TPOM. (Fig. 7b) 


\section{References}

[1] L. M. Herzig, I. Elamri, H. Schwalbe, J. Wachtveitl, Phys. Chem. Chem. Phys. 2017, 19, 14835-14844.

[2] I. Elamri, M. Heumüller, L. M. Herzig, E. Stirnal, J. Wachtveitl, E. M. Schuman, H. Schwalbe, ChemBioChem 2018, DOI 10.1002/cbic.201800408.

[3] T. Furuta, S. S. H. Wang, J. L. Dantzker, T. M. Dore, W. J. Bybee, E. M. Callaway, W. Denk, R. Y. Tsien, Proc. Natl. Acad. Sci. U. S. A. 1999, 96, 1193-1200.

[4] M. Noguchi, M. Skwarczynski, H. Prakash, S. Hirota, T. Kimura, Y. Hayashi, Y. Kiso, Bioorganic Med. Chem. 2008, 16, 5389-5397.

[5] L. Fournier, I. Aujard, T. Le Saux, S. Maurin, S. Beaupierre, J. B. Baudin, L. Jullien, Chem. - A Eur. J. 2013, 19, 17494-17507. 